Y-12

NATIONAL SECURITY COMPLEX

\section{Y-12 GROUNDWATER PROTECTION PROGRAM GROUNDWATER AND SURFACE WATER SAMPLING AND ANALYSIS PLAN FOR CALENDAR YEAR 2005}

\author{
September 2004
}

Prepared by

ELVADO ENVIRONMENTAL LLC Under Subcontract No. 4300030332

for the

Environmental Compliance Department Environment, Safety, and Health Division Y-12 National Security Complex Oak Ridge, Tennessee 37831

Managed by

BWXT Y-12, L.L.C.

for the U.S. DEPARTMENT OF ENERGY under contract No. DE-AC05-00OR22800 


\section{DISCLAIMER}

This report was prepared as an account of work sponsored by an agency of the United States Government. Neither the United States Government nor any agency thereof, nor any of their employees, makes any warranty, express or implied, or assumes any legal liability or responsibility for the accuracy, completeness, or usefulness of any information, apparatus, product, or process disclosed, or represents that its use would not infringe privately owned rights. Reference herein to any specific commercial product, process, or service by trade name, trademark, manufacturer, or otherwise, does not necessarily constitute or imply its endorsement, recommendation, or favoring of the United States Government or any agency thereof. The views and opinions of authors expressed herein do not necessarily state or reflect those of the United States Government or any agency thereof. 


\title{
Y-12 GROUNDWATER PROTECTION PROGRAM GROUNDWATER AND SURFACE WATER SAMPLING AND ANALYSIS PLAN \\ FOR CALENDAR YEAR 2005
}

September 2004

Prepared by

ELVADO ENVIRONMENTAL LLC

Under Subcontract No. 4300030332

for the

Environmental Compliance Department Environment, Safety, and Health Division Y-12 National Security Complex

Oak Ridge, Tennessee 37831

\author{
Managed by \\ BWXT Y-12, L.L.C. \\ for the U.S. DEPARTMENT OF ENERGY \\ under contract No. DE-AC05-00OR22800
}




\section{TABLE OF CONTENTS}

$\underline{\text { Section }}$

Page

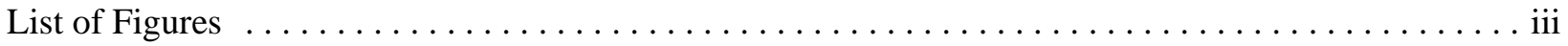

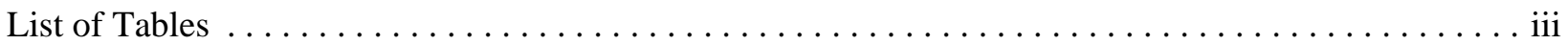

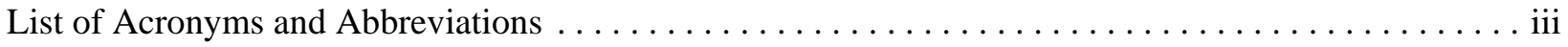

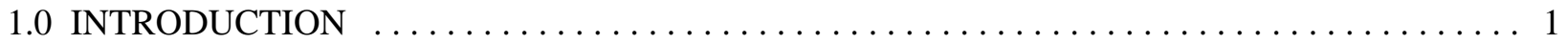

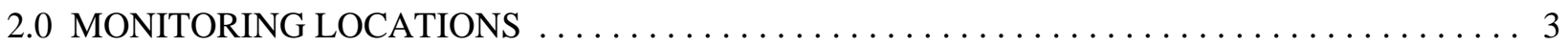

3.0 FIELD MEASUREMENTS AND ANALYTICAL PARAMETERS $\ldots \ldots \ldots \ldots \ldots \ldots \ldots \ldots$

4.0 SAMPLE PLANNING, COLLECTION, AND HANDLING $\ldots \ldots \ldots \ldots \ldots \ldots \ldots \ldots \ldots 7$

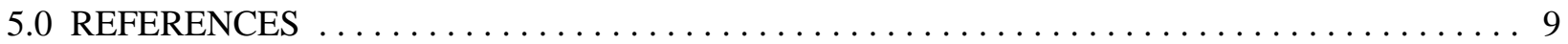

\section{APPENDICES:}

A FIGURES

B TABLES

C SAMPLING PRIORITY SCORES

D LABORATORY REQUIREMENTS

(Bottle Lists, Holding Times, Turnaround Times, Elevated Minimum Detectable Activity)

E ADDENDA TO THE CY 2005 SAMPLING AND ANALYSIS PLAN

F CY 2005 GROUNDWATER MONITORING SCHEDULES 


\section{List of Figures}

Figure

Page

A.1 Hydrogeologic regimes at the Y-12 National Security Complex $\ldots \ldots \ldots \ldots \ldots \ldots \ldots$ A-1

A.2 CY 2005 sampling locations in the Bear Creek Hydrogeologic Regime $\ldots \ldots \ldots \ldots \ldots$. . . . .

A.3 CY 2005 sampling locations in the Chestnut Ridge Hydrogeologic Regime . . . . . . . . . . A-3

A.4 CY 2005 sampling locations in the Upper East Fork Poplar Creek

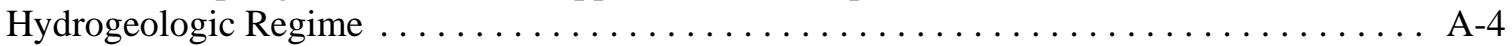

A.5 Westbay ${ }^{\mathrm{TM}}$ monitoring system sampling port depths in well GW-722 $\ldots \ldots \ldots \ldots \ldots \ldots$ A-5

A.6 CY 2005 surface water sampling locations north of Pine Ridge $\ldots \ldots \ldots \ldots \ldots \ldots \ldots$. . . . A-6

\section{List of Tables}

Table

Page

B.1 Sampling sequence, frequency, and analytical parameters for groundwater and surface water monitoring during CY $2005 \ldots \ldots \ldots \ldots \ldots \ldots \ldots \ldots \ldots \ldots \ldots \ldots \ldots$

B.2 Field measurements and analytes that comprise the elementary parameter groups for CY 2005 groundwater and surface water samples $\ldots \ldots \ldots \ldots \ldots \ldots \ldots \ldots \ldots$. $\ldots \ldots \ldots$

\section{List of Acronyms and Abbreviations}

ACO

Bear Creek Regime

BWXT

Chestnut Ridge Regime

CY

DOE

East Fork Regime

GWPP

REDOX

SPS

$\mathrm{Y}-12$
Analytical Chemistry Organization

Bear Creek Hydrogeologic Regime

BWXT Y-12, L.L.C.

Chestnut Ridge Hydrogeologic Regime

calendar year

U.S. Department of Energy

Upper East Fork Poplar Creek Hydrogeologic Regime

Groundwater Protection Program

oxidation-reduction potential

sampling priority score

Y-12 National Security Complex 


\subsection{INTRODUCTION}

This plan provides a description of the groundwater and surface water quality monitoring activities planned for calendar year (CY) 2005 at the U.S. Department of Energy (DOE) Y-12 National Security Complex (Y-12) that will be managed by the Y-12 Groundwater Protection Program (GWPP). Groundwater and surface water monitoring performed by the GWPP during CY 2005 will be in accordance with DOE Order 540.1 requirements and the following goals:

- to maintain surveillance of existing and potential groundwater contamination sources;

- to provide for the early detection of groundwater contamination and determine the quality of groundwater and surface water where contaminants are most likely to migrate beyond the Oak Ridge Reservation property line;

- to identify and characterize long-term trends in groundwater quality at Y-12; and

- to provide data to support decisions concerning the management and protection of groundwater resources.

Groundwater and surface water monitoring during CY 2005 will be performed primarily in three hydrogeologic regimes at Y-12: the Bear Creek Hydrogeologic Regime (Bear Creek Regime), the Upper East Fork Poplar Creek Hydrogeologic Regime (East Fork Regime), and the Chestnut Ridge Hydrogeologic Regime (Chestnut Ridge Regime). The Bear Creek and East Fork regimes are located in Bear Creek Valley, and the Chestnut Ridge Regime is located south of Y-12 (Figure A.1). Additional surface water monitoring will be performed north of Pine Ridge, along the boundary of the Oak Ridge Reservation (Figure A.1).

Modifications to the CY 2005 monitoring program may be necessary during implementation. Changes in programmatic requirements may alter the analytes specified for selected monitoring wells or may add or remove wells from the planned monitoring network. All modifications to the monitoring program will be approved by the Y-12 GWPP manager and documented as addenda to this sampling and analysis plan. 


\subsection{MONITORING LOCATIONS}

The monitoring locations to be sampled by the Y-12 GWPP during CY 2005 (Table B.1) are in two basic groups: (1) a fixed group of 43 monitoring wells, five springs, and 11 surface water stations that are sampled semiannually every year to address specific requirements of DOE Order surveillance monitoring; and (2) a group of supplemental monitoring wells to augment DOE Order surveillance monitoring, selected in accordance with the Y-12 GWPP Monitoring Optimization Plan (BWXT Y-12, L.L.C. [BWXT] 2003a). The monitoring optimization plan describes the technical approach implemented by the Y-12 GWPP to focus available resources on the monitoring wells at Y-12 which provide the most useful water quality monitoring data. The relative priority for sampling the supplemental wells is expressed by the annual sampling priority score (SPS). The SPS has been determined for each well granted active status (BWXT 2003a) that is not scheduled for sampling during CY 2005 by other organizations that share data with the Y-12 GWPP. Points for each well are scored for: location (the Bear Creek Regime is the focus for CY 2005); sampling history (includes the total number of samples collected and the length of time since the most recent sample); principal contaminant concentrations; and contaminant concentration trends. The SPS is the sum of these points. By sorting in descending SPS order, the wells are ranked to prioritize for sample collection. The CY 2005 SPS ranking, showing the 41 supplemental wells selected for sample collection, are provided in Appendix C.

The Y-12 GWPP monitoring network for CY 2005 includes 100 monitoring locations (Table B.1): 67 located in the Bear Creek Regime (Figure A.2), five located in the Chestnut Ridge Regime (Figure A.3), 23 located in the East Fork Regime (Figure A.4), and five located north of Pine Ridge (Figure A.5). Groundwater samples will be collected from a total of 84 monitoring wells, including 61 wells in the Bear Creek Regime (Figure A.2) and 23 wells in the East Fork Regime (Figure A.4). Well GW-722, located in the East Fork Regime, contains a Westbay ${ }^{\mathrm{TM}}$ multiport sampling system and is scheduled for sample collection from 10 ports at different depths in the well (Figure A.5). Samples of groundwater discharging from five natural springs will be collected during CY 2005, including three springs (SS-1, SS-4, and SS-5) in the Bear Creek Regime (Figure A.2) and two springs (SCR2.1SP and SCR2.2SP) in the Chestnut Ridge Regime (Figure A.3). Surface water samples will be collected from a total of 11 sampling locations during CY 2005, including three locations in the Bear Creek Regime, three locations in the Chestnut Ridge Regime, and five locations north of Pine Ridge. In the Bear Creek Regime, samples will be collected from Bear Creek at two sampling stations located from about 0.5 to 4.5 kilometers upstream of the confluence of Bear Creek and East Fork Poplar Creek (BCK-00.63 and BCK-04.55), and from one sampling station along a northern tributary (NT-01) to Bear Creek (Figure A.2). The tributaries located in the Chestnut Ridge Regime have been numbered from west to east (SCR1 through SCR5), and surface water samples will be collected from three of the tributaries at stations (SCR1.5SW, SCR3.5SW, and S17 [located in SCR5]) located along the north side of Bethel Valley Road (Figure A.3). The surface water sampling locations north of Pine Ridge include three tributaries (NPR07.0SW, NPR12.0SW, and NPR23.0SW) near the Scarboro Community and two locations (GHK2.51ESW and GHK2.51WSW) near Country Club Estates (Figure A.6). 


\subsection{FIELD MEASUREMENTS AND ANALYTICAL PARAMETERS}

Before collecting samples at each monitoring location, field personnel will record (on Field Data Sheets) the following field measurements (Table B.2):

- static water level in monitoring wells (excluding wells equipped with a Westbay ${ }^{\mathrm{TM}}$ multiport sampling system);

- $\mathrm{pH}$;

- water temperature;

- conductivity;

- dissolved oxygen; and

- oxidation-reduction potential (REDOX)

Field measurements of REDOX will not be obtained for sampling ports of monitoring wells equipped with a Westbay ${ }^{\mathrm{TM}}$ multiport sampling system.

For this Sampling and Analysis Plan, specific analytes are grouped by analytical method or by type (e.g., trace metals) and referenced as parameter groups (Table B.1 and Table B.2). In addition to field measurements, all groundwater and surface water samples will be analyzed for the following suite of parameters (identified as the Standard Administrative Parameter Group):

- miscellaneous laboratory analytes (turbidity, total suspended solids and total dissolved solids);

- major anions;

- trace metals (includes major cations);

- a comprehensive suite of organic compounds; and

- gross alpha and gross beta activity.

In addition to the analytes included in the Standard Administrative Parameter Group, samples from selected locations will be analyzed for specific radionuclides. Some of these analyses will supplement gross alpha and/or gross beta activity results, especially in cases where the gross activity reporting limits are elevated from interferences caused a by high dissolved solid content of the groundwater sample (see Appendix D). Additionally, determining the weight percent of uranium-235 (Table B.1 and Table B.2) is required to meet waste acceptance criteria for purge water disposal. 


\subsection{SAMPLE PLANNING, COLLECTION, AND HANDLING}

The monitoring wells, springs, and surface water stations included in the GWPP monitoring network for CY 2005 are assembled into sample groups (e.g., BC-1) for sample collection, sample tracking, and data management purposes. A total of 13 sample groups are scheduled for CY 2005: seven groups for the first and third quarters, and six groups for the second and fourth quarters (Table B.1). The sampling sequence is generally from least contaminated to most contaminated location within each sampling group. A Groundwater Monitoring Schedule will be prepared for each quarterly sampling event by GWPP personnel based on Table B.1 that includes additional information necessary for field personnel to collect the required samples (e.g., management of purged groundwater).

Samples will be collected semiannually from all monitoring locations during CY 2005. As summarized below, the number of samples to be collected during each CY quarter (including conventional method samples) will range from 53 to 60, for an annual total of 226 samples.

\begin{tabular}{|c|c|c|}
\hline \multirow{2}{*}{ HYDROGEOLOGIC } & \multicolumn{2}{|c|}{ NUMBER OF SAMPLES PER QUARTER OF CY 2005 } \\
\cline { 2 - 3 } REGIME/AREA & 1st and 3rd & 2nd and 4th \\
\hline Bear Creek Regime & 45 & 26 \\
Chestnut Ridge Regime & 5 & 0 \\
East Fork Regime & 10 & 22 \\
North of Pine Ridge & 0 & 5 \\
\hline TOTAL: & $\mathbf{6 0}$ & $\mathbf{5 3}$ \\
\hline
\end{tabular}

Personnel from the Y-12 Analytical Chemistry Organization (ACO) will be responsible for collection, transportation, and chain-of-custody control of most groundwater and surface water samples. Personnel from the Environment, Safety, and Health Division will be responsible for collection, transportation and chain-ofcustody control of the groundwater samples from Westbay well GW-722. Based on the analytical parameters for CY 2005 (Table B.1 and Table B.2), ACO personnel will prepare bottle lists that specify the sample container type, size, preservative, and the laboratory test identification needed for each sampling location (see Appendix D). Sample collection will be performed in accordance with the most recent version of operating procedures for obtaining groundwater samples (BWXT 2002a, BWXT 2002b, BWXT 2004a, and BWXT 2004b) and surface water samples (BWXT 2002c). All field and laboratory activities will be performed in accordance with applicable requirements of the Y-12 Integrated Safety Management System.

Groundwater samples will be collected for the low-flow minimal drawdown method (low-flow method) from all monitoring wells during CY 2005, unless otherwise specified (Table B.1). For the low-flow method, a bladder pump is permanently installed in each well that is scheduled for sample collection. If well construction prevents permanent installation (e.g., flush-mounted wells), then the pump and tubing will be installed at least 24 hours before sample collection and will be removed when sampling is completed. In accordance with the groundwater sampling procedure for the low-flow method (BWXT 2004a), groundwater is purged, and subsequently sampled, from the well at a flow rate ( $<300$ milliliters per minute) which ensures minimal drawdown of the static water level, therefore isolating the stagnant water column above the intake of the pump. Groundwater samples are collected from a well after the water level is in steady-state drawdown $(<0.1 \mathrm{ft}$ over a 15-minute interval) and field parameters ( $\mathrm{pH}$, conductivity, water temperature, REDOX, and dissolved oxygen) have stabilized (minimal variation over four consecutive readings). 
Samples from selected monitoring wells will be collected by the "conventional" sampling method using a gas piston Bennett ${ }^{\circledR}$ pump. The conventional sampling method, which was used to collect all groundwater samples through September 1997, involves removing three well-volumes of groundwater from a well (or purging the well dry) at about 5,600 milliliters per minute (much higher than the purge rate for low-flow) before collecting samples. Although the analytical results for the majority of wells do not show a distinct response to the change in method from the conventional to low-flow sampling, the analytical results for some wells potentially exhibit a clear response (BWXT 2002d and BWXT 2003b). To further investigate this phenomenon, samples will be obtained using the conventional method the day after collecting samples using the low-flow method at four wells (GW-071, GW-072, GW-624, and GW-626) in the Bear Creek Regime (Table B.1).

Groundwater sampling and pressure profiling using a Westbay ${ }^{\mathrm{TM}}$ multiport sampling system at well GW-722 in the East Fork Regime will be performed in accordance with the operating procedures (BWXT 2002a and 2002b). The groundwater samples from each sampling port (Figure A.5) will be collected in 250-milliliter nonvented stainless steel Westbay ${ }^{\mathrm{TM}}$ sample collection bottles filled at the designated depth in the well. Once filled, the bottles will be raised to the surface and the groundwater will be transferred to laboratory sample containers. The sample collection bottles will be lowered, filled, and retrieved as many times as needed to completely fill the laboratory sample bottles. Groundwater in the first sample collection bottles retrieved from each sampling port will be used as a "formation rinse" to obtain field measurements and to condition the sample collection bottle for each zone.

Unfiltered samples will be collected from all of the monitoring locations during CY 2005. Because analytical results have shown that the conventional sampling method may provide samples that are more turbid than the low-flow sampling method, filtered samples will also be collected from the three wells specified for conventional sampling (Table B.1). These samples will be filtered in the field using a 0.45 -micron filter and analyzed for dissolved trace metal concentrations.

In addition to the groundwater and surface water samples, field blanks and equipment rinsate samples will be collected at the frequencies and analyzed for the parameter groups specified on Table B.1. Field blank samples will be collected from at least $10 \%$ of the sample groups. Therefore, one field blank will be collected during each quarter of CY 2005: in the Bear Creek Regime during the first and third quarters and in the East Fork Regime during the second and fourth quarters. Equipment rinsate samples will be collected from Westbay well GW-722 and from selected wells scheduled for conventional sampling (Table B.1). The rinsate sample will be collected immediately after field-cleaning the sampling equipment used to collect samples from the last sampling port (GW-722-17) or the last well sampled in a sample group (GW-624 and GW-071).

Trip blank samples, field duplicate samples, and laboratory quality assurance samples will be prepared and analyzed as specified in the Quality Assurance Plan for the Analytical Chemistry Organization (BWXT 2003c) using applicable analytical procedures. Trip blank samples will be prepared for each cooler used to transport samples for volatile organic analyses. Duplicate samples will be collected from at least $10 \%$ of the sampling locations. A total of 26 field duplicate samples will be collected during CY 2005, including 14 in the Bear Creek Regime, two in the Chestnut Ridge Regime, eight in the East Fork Regime, and two from surface water stations located north of Pine Ridge (Table B.1).

All groundwater and surface water samples will be relinquished under chain-of-custody control to the appropriate Y-12 ACO laboratory that will perform the analyses. The Y-12 ACO laboratories will perform each analyses within established holding times and deliver results within established turnaround times (see Appendix D). 


\subsection{REFERENCES}

BWXT Y-12, L.L.C. 2002a. Groundwater Sampling of Westbay ${ }^{\mathrm{TM}}$ Monitoring System Instrumented Wells. BWXT Y-12, L.L.C. Management Requirement prepared by the Environment, Safety, and Health Organization (Y50-71-018, Rev.1).

BWXT Y-12, L.L.C. 2002b. Pressure Profiling of Wells Equipped with Westbay ${ }^{\mathrm{TM}}$ Monitoring System Instrumentation. BWXT Y-12, L.L.C. Management Requirement prepared by the Environment, Safety, and Health Organization (Y50-71-019, Rev.1).

BWXT Y-12, L.L.C. 2002c. Liquid Grab Sampling. BWXT Y-12, L.L.C. Management Requirement prepared by the Environment, Safety, and Health Organization (Y50-71-005, Rev. 1.1).

BWXT Y-12, L.L.C. 2002d. Calendar Year 2001 Groundwater Monitoring Data Evaluation Report for the U.S. Department of Energy Y-12 National Security Complex, Oak Ridge, Tennessee. Prepared by AJA Technical Services, Inc. (Y/SUB/02-012529/2).

BWXT Y-12, L.L.C. 2003a. Y-12 Groundwater Protection Program Monitoring Optimization Plan for Groundwater Monitoring Wells at the U.S. Department of Energy Y-12 National Security Complex, Oak Ridge, Tennessee. Prepared by Elvado Environmental LLC (Y/SUB/03-021559/2).

BWXT Y-12, L.L.C. 2003b. Calendar Year 2002 Groundwater Monitoring Data Evaluation Report for the U.S. Department of Energy Y-12 National Security Complex, Oak Ridge, Tennessee. Prepared by Elvado Environmental LLC (Y/SUB/03-021559/3).

BWXT Y-12, L.L.C. 2003c. Quality Assurance Plan for the Analytical Chemistry Organization. Prepared by the Analytical Chemistry Organization (Y/P65-9006, Rev. M).

BWXT Y-12, L.L.C. 2004a. Groundwater Sampling. Oak Ridge Y-12 National Security Complex Procedure prepared by the Environment, Safety, and Health Organization (Y50-71-016).

BWXT Y-12, L.L.C. 2004b. Measurement of Static Water Level Elevation. Oak Ridge Y-12 National Security Complex Procedure prepared by the Environment, Safety, and Health Organization (Y5071-015).

U.S. Environmental Protection Agency. 1983. Methods for Chemical Analysis of Water and Wastes.

U.S. Environmental Protection Agency. 1996. Test Methods for Evaluating Solid Waste Physical/Chemical Methods. 
APPENDIX A

\section{FIGURES}




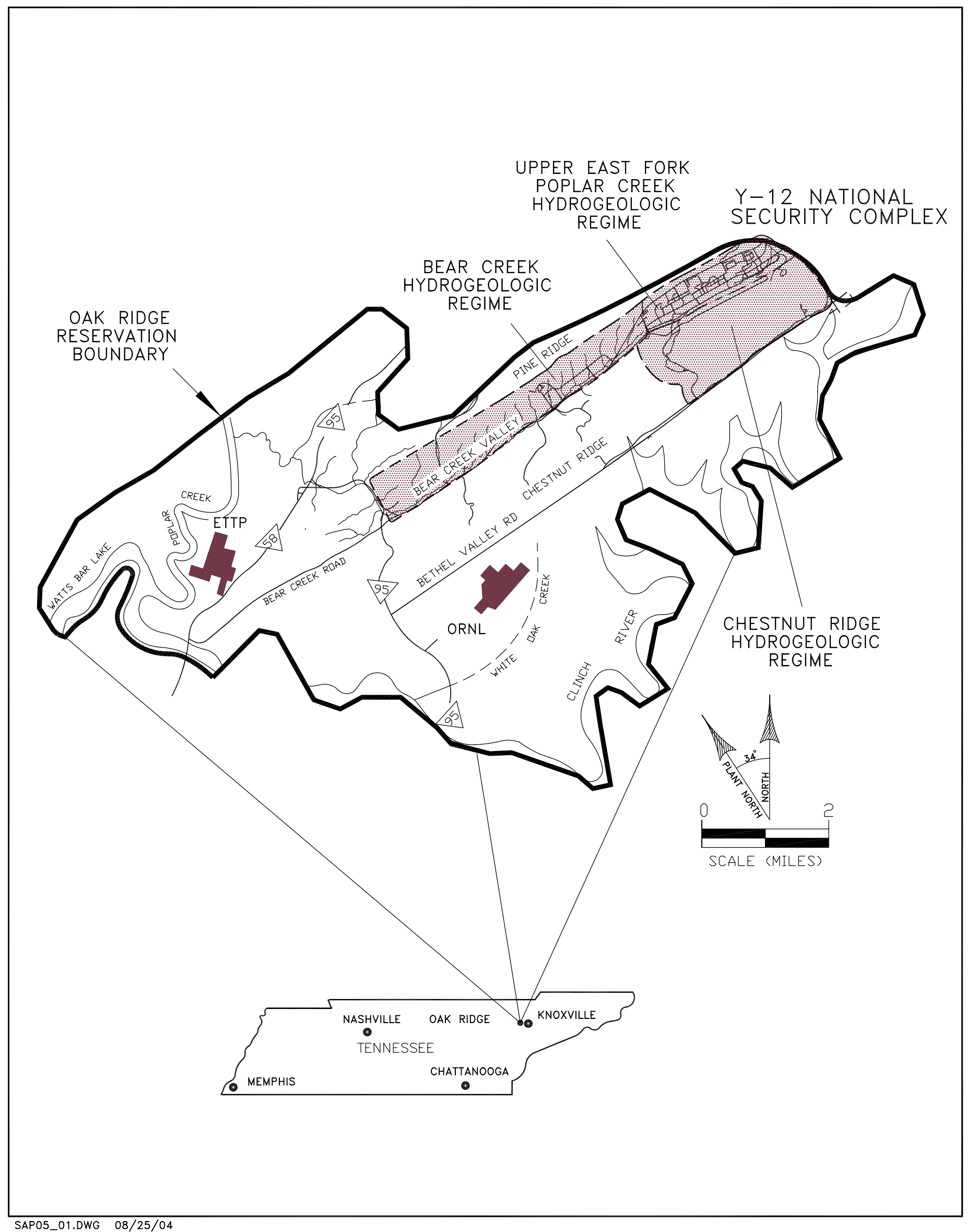

Fig. A.1. Hydrogeologic regimes at the Y-12 National Security Complex. 


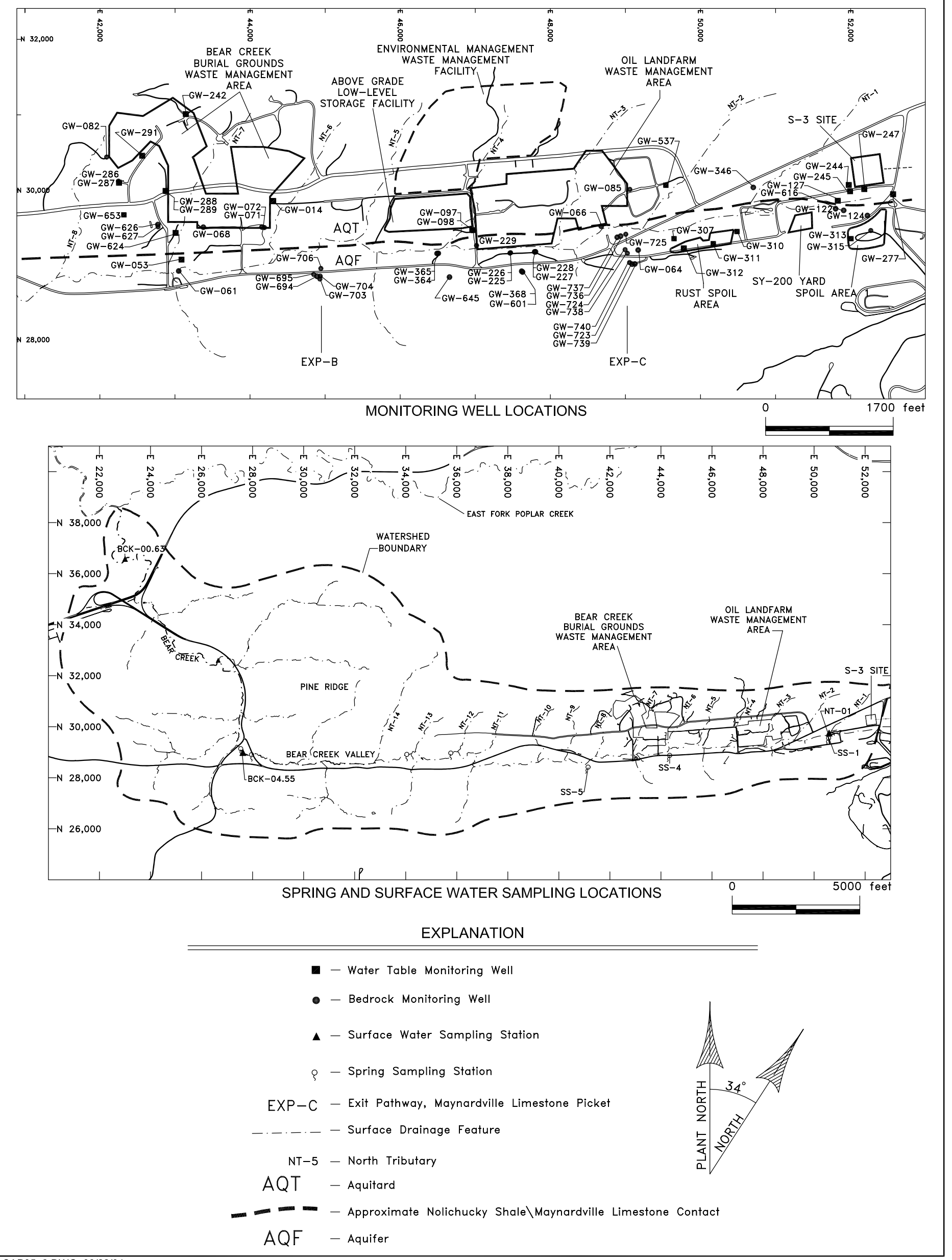

Fig. A.2. CY 2005 sampling locations in the Bear Creek Hydrogeologic Regime. 


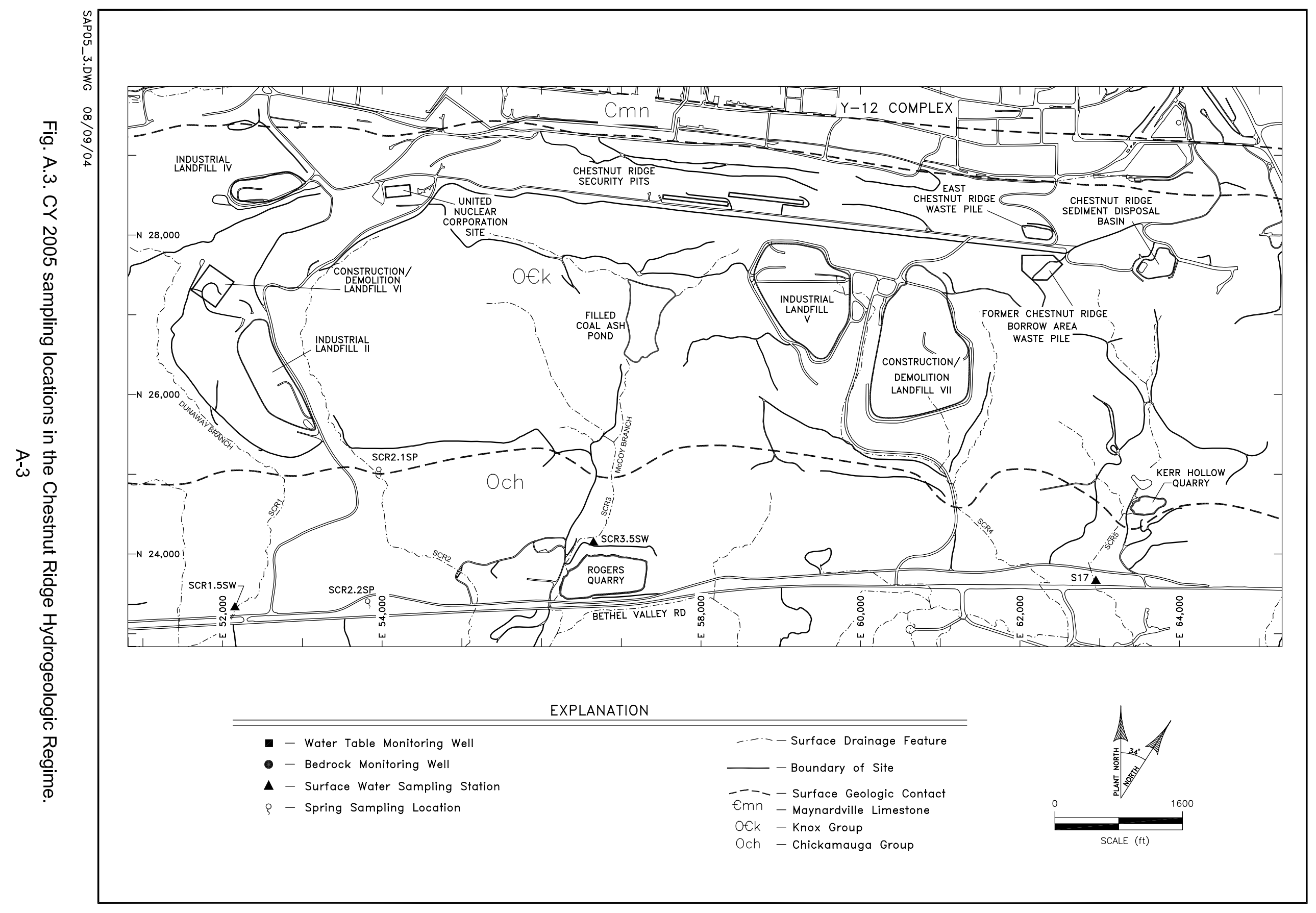




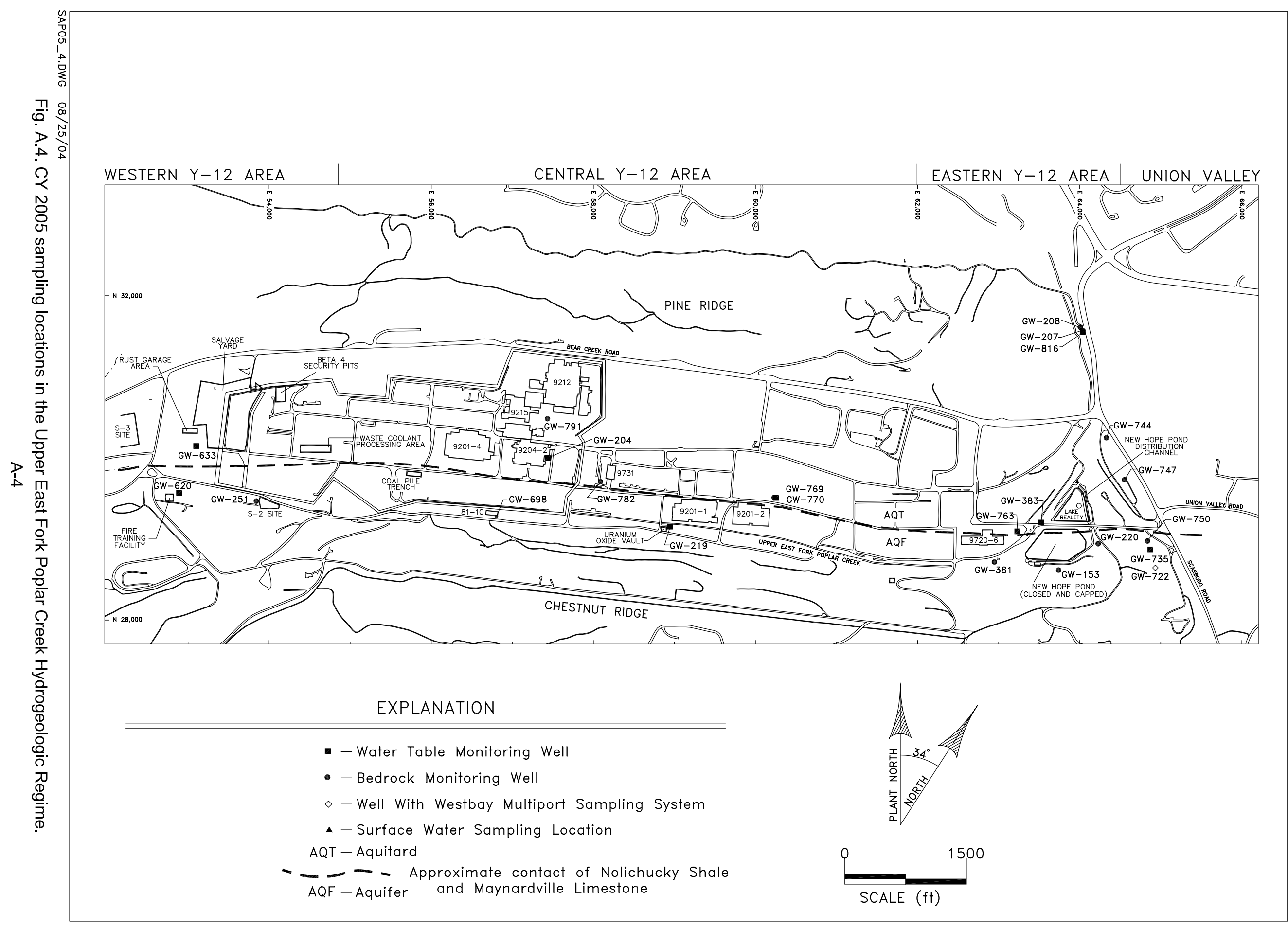




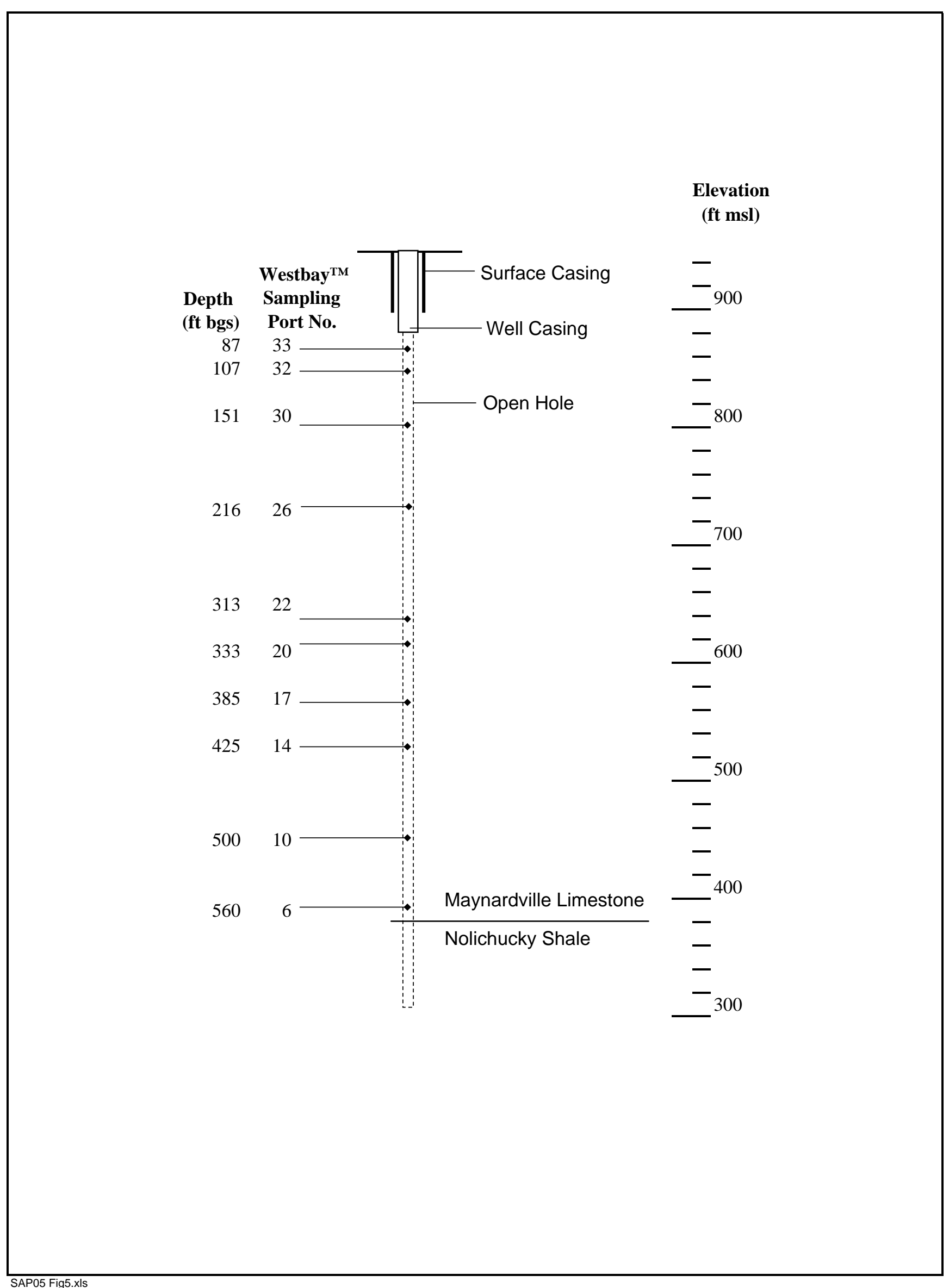

Fig. A.5. Westbay ${ }^{\mathrm{TM}}$ monitoring system sampling port depths in well GW-722. 


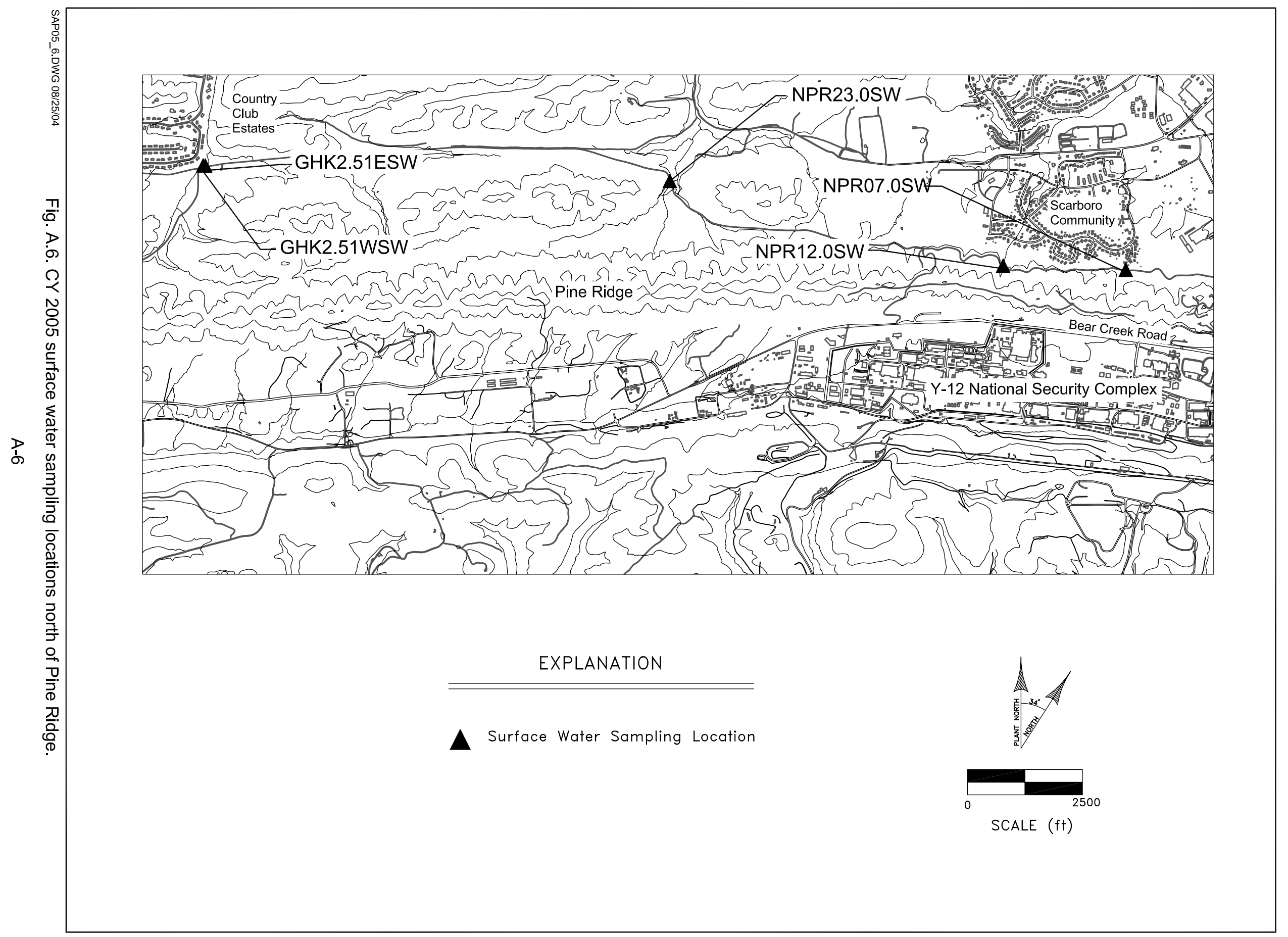


APPENDIX B

TABLES 
Table B.1. Sampling sequence, frequency, and analytical parameters for groundwater and surface water monitoring during CY 2005

\begin{tabular}{|c|c|c|c|c|c|c|}
\hline $\begin{array}{l}\text { Sample } \\
\text { Group }{ }^{1}\end{array}$ & Location $^{2}$ & $\begin{array}{c}\text { Sampling } \\
\text { Point }^{3}\end{array}$ & Duplicate $^{4}$ & $\begin{array}{l}\text { Monitoring } \\
\text { Driver }^{5}\end{array}$ & Supplement ${ }^{6}$ & $\begin{array}{c}\text { Parameter } \\
\text { Groups }^{7}\end{array}$ \\
\hline \multicolumn{7}{|c|}{ Bear Creek Hydrogeologic Regime } \\
\hline \multirow{14}{*}{$\begin{array}{l}\text { BC-1 } \\
\text { (Q1, Q3) }\end{array}$} & EXP-B & GW-694 & Q1 & SMP & $\mathrm{Y}$ & STD, RAD(13) \\
\hline & EXP-B & GW-695 & & SMP & & STD, RAD(13) \\
\hline & EXP-B & GW-703 & & SMP & & STD, RAD(13) \\
\hline & EXP-B & GW-704 & & SMP & & STD, RAD(13) \\
\hline & EXP-B & GW-706 & & SMP & & STD, RAD(13) \\
\hline & EXP-C & GW-064 & & SMP & $\mathrm{Y}$ & STD, RAD(13) \\
\hline & EXP-C & GW-723 & & SMP & $\mathrm{Y}$ & STD \\
\hline & EXP-C & GW-736 & & SMP & $\mathrm{Y}$ & $\mathrm{STD}, \operatorname{RAD}(3,12,13)$ \\
\hline & EXP-C & GW-737 & & SMP & $\mathrm{Y}$ & STD, RAD(13) \\
\hline & EXP-C & GW-739 & Q3 & SMP & $\mathrm{Y}$ & STD \\
\hline & EXP-C & GW-740 & & SMP & & STD \\
\hline & EXP-C & GW-738 & & SMP & & STD, RAD(13) \\
\hline & EXP-C & GW-724 & & SMP & & STD \\
\hline & EXP-C & GW-725 & & SMP & & STD, RAD(13) \\
\hline \multirow{10}{*}{$\begin{array}{l}\text { BC-2 } \\
\text { (Q1, Q3) }\end{array}$} & RS & GW-311 & & SMP & & STD \\
\hline & SPI & GW-313 & & SMP & $\mathrm{Y}$ & STD, RAD(12,13) \\
\hline & SPI & GW-315 & & SMP & & STD, RAD(13) \\
\hline & $\mathrm{RS}$ & GW-307 & & SMP & $\mathrm{Y}$ & STD, RAD $(12,13)$ \\
\hline & $\mathrm{RS}$ & GW-312 & & SMP & $\mathrm{Y}$ & STD, RAD(13) \\
\hline & RS & GW-310 & Q1 & SMP & $\mathrm{Y}$ & STD, RAD(13) \\
\hline & S3 & GW-124 & & SMP & $\mathrm{Y}$ & STD, RAD $(12,13)$ \\
\hline & S3 & GW-616 & & SMP & $\mathrm{Y}$ & STD, RAD(3) \\
\hline & S3 & GW-122 & Q3 & SMP & $\mathrm{Y}$ & STD, RAD $(12,13)$ \\
\hline & S3 & GW-277 & & SMP & $\mathrm{Y}$ & $\operatorname{STD}, \operatorname{RAD}(3,12,13)$ \\
\hline \multirow{6}{*}{$\begin{array}{l}\text { BC-3 } \\
\text { (Q1, Q3) }\end{array}$} & EXP-SW & BCK-00.63 & Q1 & EXP & & STD \\
\hline & EXP-SW & BCK-04.55 & & EXP & & STD \\
\hline & EXP-SW & SS-5 & & EXP & & STD \\
\hline & EXP-SW & SS-4 & Q3 & EXP & & STD \\
\hline & EXP-SW & SS-1 & & EXP & & STD \\
\hline & EXP-SW & NT-01 & & EXP & & STD \\
\hline
\end{tabular}


Table B.1 (continued)

\begin{tabular}{|c|c|c|c|c|c|c|}
\hline $\begin{array}{l}\text { Sample } \\
\text { Group }{ }^{1}\end{array}$ & Location $^{2}$ & $\begin{array}{c}\text { Sampling } \\
\text { Point }^{3}\end{array}$ & Duplicate $^{4}$ & $\begin{array}{c}\text { Monitoring } \\
\text { Driver }^{5}\end{array}$ & Supplement ${ }^{6}$ & $\begin{array}{l}\text { Parameter } \\
\text { Groups }^{7}\end{array}$ \\
\hline \multirow{8}{*}{$\begin{array}{l}\text { BC-4 } \\
\text { (Q1, Q3) }\end{array}$} & OLF & GW-097 & Q1 & SMP & $\mathrm{Y}$ & STD \\
\hline & OLF & GW-098 & & SMP & & STD, RAD(13) \\
\hline & OLF & GW-645 & & SMP & $\mathrm{Y}$ & STD, RAD(13) \\
\hline & OLF & GW-364 & & SMP & $\mathrm{Y}$ & STD, RAD(13) \\
\hline & OLF & GW-365 & & SMP & $\mathrm{Y}$ & STD \\
\hline & OLF & GW-066 & Q3 & SMP & $\mathrm{Y}$ & STD, RAD(13) \\
\hline & OLF & GW-085 & & SMP & & STD \\
\hline & OLF & GW-537 & & SMP & & STD, RAD $(3,13)$ \\
\hline \multirow{8}{*}{$\begin{array}{l}\text { BC-5 } \\
\text { (Q1, Q3) }\end{array}$} & OLF & GW-227 & Q1 & SMP & $\mathrm{Y}$ & $\operatorname{STD}, \operatorname{RAD}(3,12,13)$ \\
\hline & OLF & GW-228 & & SMP & $\mathrm{Y}$ & STD, RAD(13) \\
\hline & OLF & GW-368 & Q3 & SMP & $\mathrm{Y}$ & STD, RAD(13) \\
\hline & OLF & GW-601 & & SMP & $\mathrm{Y}$ & STD, RAD(12) \\
\hline & OLF & GW-226 & & SMP & & STD, RAD(13) \\
\hline & OLF & GW-225 & & SMP & & STD, RAD(13) \\
\hline & OLF & GW-229 & & SMP & & STD, RAD(13) \\
\hline & \multicolumn{4}{|c|}{ FIELD BLANK } & & $\operatorname{VOC}(1)$ \\
\hline \multirow{14}{*}{$\begin{array}{l}\text { BC-6 } \\
\text { (Q2, Q4) }\end{array}$} & BG & GW-286 & Q2 & SMP & $\mathrm{Y}$ & STD \\
\hline & BG & GW-287 & & SMP & $\mathrm{Y}$ & STD \\
\hline & BG & GW-653 & & SMP & & STD \\
\hline & BG & GW-242 & & SMP & $\mathrm{Y}$ & STD, RAD(3) \\
\hline & BG & GW-288 & & SMP & $\mathrm{Y}$ & STD \\
\hline & BG & GW-289 & & SMP & $\mathrm{Y}$ & STD \\
\hline & $\mathrm{BG}$ & GW-291 & $\mathrm{Q} 4$ & SMP & $\mathrm{Y}$ & STD \\
\hline & $\mathrm{BG}$ & GW-627 & & SMP & & STD \\
\hline & BG & GW-626 & & SMP & $\mathrm{Y}$ & STD \\
\hline & $\mathrm{BG}$ & GW-626 (C) & & SMP & $\mathrm{Y}$ & $\mathrm{STD} / \mathrm{F}$ \\
\hline & BG & GW-082 & & SMP & & STD \\
\hline & BG & GW-624 & & & $\mathrm{Y}$ & STD, RAD(13) \\
\hline & $\mathrm{BG}$ & GW-624 (C) & & & $\mathrm{Y}$ & $\mathrm{STD} / \mathrm{F},(\mathrm{RAD} 13)$ \\
\hline & \multicolumn{4}{|c|}{ RINSATE SAMPLE (GW-624) } & & STD \\
\hline
\end{tabular}


Table B.1 (continued)

\begin{tabular}{|c|c|c|c|c|c|c|}
\hline $\begin{array}{l}\text { Sample } \\
\text { Group }{ }^{1}\end{array}$ & Location $^{2}$ & $\begin{array}{c}\text { Sampling } \\
\text { Point }^{3}\end{array}$ & Duplicate $^{4}$ & $\begin{array}{c}\text { Monitoring } \\
\text { Driver }^{5}\end{array}$ & Supplement ${ }^{6}$ & $\begin{array}{l}\text { Parameter } \\
\text { Groups }^{7}\end{array}$ \\
\hline \multirow{14}{*}{$\begin{array}{l}\text { BC-7 } \\
\text { (Q2, Q4) }\end{array}$} & BG & GW-053 & & SMP & $\mathrm{Y}$ & STD, RAD(13) \\
\hline & BG & GW-061 & & SMP & $\mathrm{Y}$ & $\operatorname{STD}, \operatorname{RAD}(3,12,13)$ \\
\hline & BG & GW-072 & & SMP & $\mathrm{Y}$ & STD \\
\hline & BG & GW-072 (C) & & SMP & $\mathrm{Y}$ & $\mathrm{STD} / \mathrm{F}$ \\
\hline & BG & GW-068 & & SMP & $\mathrm{Y}$ & STD, RAD(13) \\
\hline & BG & GW-014 & & SMP & $\mathrm{Y}$ & STD, RAD(13) \\
\hline & BG & GW-071 & & SMP & $\mathrm{Y}$ & STD \\
\hline & BG & GW-071 (C) & & SMP & $\mathrm{Y}$ & $\mathrm{STD} / \mathrm{F}$ \\
\hline & S3 & GW-346 & & SMP & $\mathrm{Y}$ & STD, RAD(3,12,13) \\
\hline & S3 & GW-127 & & SMP & $\mathrm{Y}$ & STD, RAD(3,12,13) \\
\hline & S3 & GW-244 & & SMP & $\mathrm{Y}$ & $\operatorname{STD}, \operatorname{RAD}(3,12,13)$ \\
\hline & S3 & GW-247 & & SMP & $\mathrm{Y}$ & $\mathrm{STD}, \operatorname{RAD}(3,12,13)$ \\
\hline & S3 & GW-245 & & SMP & $\mathrm{Y}$ & $\mathrm{STD}, \operatorname{RAD}(3,12,13)$ \\
\hline & \multicolumn{4}{|c|}{ RINSATE SAMPLE (GW-071) } & & STD \\
\hline \multicolumn{7}{|c|}{ Chestnut Ridge Hydrogeologic Regime } \\
\hline \multirow{5}{*}{$\begin{array}{l}\text { CR-1 } \\
\text { (Q1,Q3) }\end{array}$} & EXP-SW & SCR1.5SW & Q3 & EXP & & STD \\
\hline & EXP-SW & SCR2.1SP & & EXP & & STD \\
\hline & EXP-SW & SCR2.2SP & & EXP & & STD \\
\hline & EXP-SW & SCR3.5SW & Q1 & EXP & & STD \\
\hline & EXP-SW & S17 & & EXP & & STD \\
\hline \multicolumn{7}{|c|}{ Upper East Fork Poplar Creek Hydrogeologic Regime } \\
\hline \multirow{7}{*}{$\begin{array}{l}\text { EF-1 } \\
(\mathrm{Q} 2, \mathrm{Q} 4)\end{array}$} & FTF & GW-620 & Q2 & SMP & & STD \\
\hline & S2 & GW-251 & & SMP & & STD, RAD(13) \\
\hline & T0134 & GW-204 & & SMP & & STD, RAD(13) \\
\hline & GRID E3 & GW-782 & & SMP & & STD \\
\hline & RG & GW-633 & & SMP & & $\operatorname{STD}, \operatorname{RAD}(3,12,13)$ \\
\hline & GRID D2 & GW-791 & Q4 & SMP & & STD \\
\hline & \multicolumn{4}{|c|}{ FIELD BLANK } & & $\operatorname{VOC}(1)$ \\
\hline
\end{tabular}


Table B.1 (continued)

\begin{tabular}{|c|c|c|c|c|c|c|}
\hline $\begin{array}{l}\text { Sample } \\
\text { Group }{ }^{1}\end{array}$ & Location $^{2}$ & $\begin{array}{c}\text { Sampling } \\
\text { Point }^{3}\end{array}$ & Duplicate $^{4}$ & $\begin{array}{l}\text { Monitoring } \\
\text { Driver }^{5}\end{array}$ & Supplement ${ }^{6}$ & $\begin{array}{l}\text { Parameter } \\
\text { Groups }^{7}\end{array}$ \\
\hline \multirow{8}{*}{$\begin{array}{l}\text { EF-2 } \\
(\mathrm{Q} 2, \mathrm{Q} 4)\end{array}$} & GRID G3 & GW-770 & & SMP & & STD \\
\hline & GRID G3 & GW-769 & & SMP & & STD \\
\hline & NHP & GW-153 & & SMP & & STD, RAD(13) \\
\hline & $\mathrm{UOV}$ & GW-219 & Q2 & SMP & & STD, RAD(13) \\
\hline & GRID JP & GW-763 & & SMP & & STD \\
\hline & $\mathrm{B} 8110$ & GW-698 & & SMP & & STD \\
\hline & NHP & GW-381 & $\mathrm{Q} 4$ & SMP & & STD \\
\hline & NHP & GW-383 & & SMP & & STD \\
\hline \multirow{8}{*}{$\begin{array}{l}\text { EF-3 } \\
(\mathrm{Q} 2, \mathrm{Q} 4)\end{array}$} & EXP-SR & GW-208 & & EXP & & STD \\
\hline & EXP-SR & GW-207 & & EXP & & STD \\
\hline & EXP-SR & GW-816 & & EXP & & STD \\
\hline & GRID K1 & GW-744 & $\mathrm{Q} 2$ & EXP & & STD \\
\hline & GRID K2 & GW-747 & & EXP & & STD \\
\hline & EXP-J & GW-750 & & EXP & & STD \\
\hline & EXP-J & GW-735 & & EXP & & STD \\
\hline & NHP & GW-220 & $\mathrm{Q} 4$ & EXP & & STD \\
\hline \multirow{11}{*}{$\begin{array}{l}\text { EF-WB } \\
\text { (Q1,Q3) }\end{array}$} & EXP-J & GW-722-06 & & EXP & & STD \\
\hline & EXP-J & GW-722-30 & & EXP & & STD \\
\hline & EXP-J & GW-722-26 & & EXP & & STD \\
\hline & EXP-J & GW-722-32 & Q1 & EXP & & STD \\
\hline & EXP-J & GW-722-33 & & EXP & & STD \\
\hline & EXP-J & GW-722-10 & & EXP & & STD \\
\hline & EXP-J & GW-722-22 & & EXP & & STD \\
\hline & EXP-J & GW-722-20 & & EXP & & STD \\
\hline & EXP-J & GW-722-14 & & EXP & & STD \\
\hline & EXP-J & GW-722-17 & Q3 & EXP & & STD \\
\hline & \multicolumn{4}{|c|}{ RINSATE SAMPLE } & & STD \\
\hline
\end{tabular}


Table B.1 (continued)

\begin{tabular}{|c|c|c|c|c|c|c|}
\hline $\begin{array}{l}\text { Sample } \\
\text { Group }{ }^{1}\end{array}$ & Location $^{2}$ & $\begin{array}{c}\text { Sampling } \\
\text { Point }^{3}\end{array}$ & Duplicate $^{4}$ & $\begin{array}{c}\text { Monitoring } \\
\text { Driver }^{5}\end{array}$ & Supplement ${ }^{6}$ & $\begin{array}{c}\text { Parameter } \\
\text { Groups }^{7}\end{array}$ \\
\hline \multicolumn{7}{|c|}{ North of Pine Ridge } \\
\hline \multirow{5}{*}{$\begin{array}{l}\text { PR-1 } \\
(\mathrm{Q} 2, \mathrm{Q} 4)\end{array}$} & EXP-NPR & NPR07.0SW & Q2 & EXP & & STD \\
\hline & EXP-NPR & NPR12.0SW & & EXP & & STD \\
\hline & EXP-NPR & NPR23.0SW & & EXP & & STD \\
\hline & EXP-NPR & GHK2.51ESW & Q4 & EXP & & STD \\
\hline & EXP-NPR & GHK2.51WSW & & EXP & & STD \\
\hline
\end{tabular}

Notes:

1 Samples will be collected during the calendar year quarter as specified (e.g., Q1).

2 Bear Creek Hydrogeologic Regime

BG - Bear Creek Burial Grounds Waste Management Area

EXP-B - Exit Pathway Picket B

EXP-C - Exit Pathway Picket C

EXP-SW - Spring or Surface Water Location

OLF - Oil Landfarm Waste Management Area

RS - Rust Spoil Area

S3 - S3 Site

SPI - Spoil Area I

\section{Chestnut Ridge Hydrogeologic Regime}

EXP-SW - Spring or surface water sampling location

\section{Upper East Fork Poplar Creek Hydrogeologic Regime}

B8110 - Building 81-10

B9201-2 - Building 9201-2

CPT - Coal Pile Trench

EXP-J - Maynardville Limestone Exit Pathway Picket J

EXP-SR - Exit pathway well in the gap through Pine Ridge along Scarboro Road

FTF - Fire Training Facility

GRID - Comprehensive Groundwater Monitoring Plan Grid Location

NHP - New Hope Pond

RG - Rust Garage Area

T0134 - Underground Storage Tank 0134-U

UOV - Uranium Oxide Vault

S2 - S-2 Site

Y12 - Y-12 Complex

\section{North of Pine Ridge}

EXP-NPR - Surface water sampling station located where drainage exits the Oak Ridge Reservation 


\section{Table B.1 (continued)}

\section{Notes: (continued)}

3 BCK - Bear Creek Kilometer (surface water station)

GW - Groundwater monitoring well

GHK - Gum Hollow Kilometer (surface water station)

NPR - North of Pine Ridge (surface water station)

NT - North Tributary to Bear Creek

S17 - Surface water station in SCR5

SCR - South Chestnut Ridge (spring or surface water station)

SS - Spring sampling location: South Side of Bear Creek

(C) - A sample will be collected using the conventional sampling method the day after collecting the sample using the low-flow sampling method.

$4 \quad Q_{-} \quad$ - Field duplicate samples will be collected at these locations during the quarter specified.

5 EXP - DOE Order Exit Pathway/Perimeter Monitoring

SMP - DOE Order Surveillance Monitoring

$6 \quad \mathrm{Y}-$ Monitoring location selected to augment the fixed annual monitoring network (see Appendix C).

7 Table B.2 provides a comprehensive list of analytes, analytical methods, and the associated parameter group.

STD - Standard administrative parameter group, including the following elementary parameter groups:

FLD - Field measurements

CHEM - Miscellaneous laboratory analytes (e.g., dissolved solids) and anions

MET(1) - Metals

VOC(1) - Volatile organic compounds

RAD(1) - Gross alpha and gross beta activity

\section{Radionuclide Elementary Parameter Groups:}

RAD(3) - Uranium-234, -235, and -238

RAD(12) - Technetium-99

RAD(13) - Total uranium and weight percent of uranium-235

\section{Special Sample Elementary Parameter Group:}

STD/F - Standard administrative parameter group, plus a filtered sample for dissolved metals analyses; collected using the conventional sampling method with a 0.45 -micron filter. 
Table B.2. Field measurements and analytes that comprise the elementary parameter groups for CY 2005 groundwater and surface water samples

\begin{tabular}{|c|c|c|c|c|}
\hline $\begin{array}{c}\text { Parameter } \\
\text { Group }\end{array}$ & $\begin{array}{c}\text { Measurement or } \\
\text { Analyte }\end{array}$ & $\begin{array}{c}\text { Analytical } \\
\text { Method }^{1}\end{array}$ & $\begin{array}{c}\text { Reporting } \\
\text { Limit }^{2}\end{array}$ & Units $^{3}$ \\
\hline \multirow[t]{6}{*}{ FLD } & Depth to Water & NA & NA & $\mathrm{ft}$ \\
\hline & Water Temperature & NA & NA & centigrade \\
\hline & $\mathrm{pH}$ & NA & NA & $\mathrm{pH}$ units \\
\hline & Conductivity & NA & NA & $\mu \mathrm{mho} / \mathrm{cm}$ \\
\hline & Dissolved Oxygen & NA & NA & $\mathrm{ppm}$ \\
\hline & Oxidation-Reduction Potential & NA & NA & $\mathrm{mV}$ \\
\hline \multirow{3}{*}{$\begin{array}{c}\text { CHEM } \\
\text { (miscellaneous) }\end{array}$} & Total Dissolved Solids & EPA-160.1 & 1 & $\mathrm{mg} / \mathrm{L}$ \\
\hline & Total Suspended Solids & EPA-160.2 & 1 & $\mathrm{mg} / \mathrm{L}$ \\
\hline & Turbidity & EPA-180.1 & 0.1 & NTU \\
\hline \multirow{6}{*}{$\begin{array}{l}\text { CHEM } \\
\text { (anions) }\end{array}$} & Alkalinity - HCO3 & EPA-310.1 & 1.0 & $\mathrm{mg} / \mathrm{L}$ \\
\hline & Alkalinity - CO3 & EPA-310.1 & 1.0 & $\mathrm{mg} / \mathrm{L}$ \\
\hline & Chloride & EPA-300.0 & 0.2 & $\mathrm{mg} / \mathrm{L}$ \\
\hline & Fluoride & EPA-340.2 & 0.1 & $\mathrm{mg} / \mathrm{L}$ \\
\hline & Nitrate (as Nitrogen) & EPA-300.0 & 0.028 & $\mathrm{mg} / \mathrm{L}$ \\
\hline & Sulfate & EPA-300.0 & 0.25 & $\mathrm{mg} / \mathrm{L}$ \\
\hline \multirow[t]{22}{*}{ MET(1) } & Aluminum & SW846-6010B & 0.2 & $\mathrm{mg} / \mathrm{L}$ \\
\hline & Antimony & EPA-200.8 & 0.0025 & $\mathrm{mg} / \mathrm{L}$ \\
\hline & Arsenic & EPA-200.8 & 0.005 & $\mathrm{mg} / \mathrm{L}$ \\
\hline & Barium & SW846-6010B & 0.004 & $\mathrm{mg} / \mathrm{L}$ \\
\hline & Beryllium & SW846-6010B & 0.0005 & $\mathrm{mg} / \mathrm{L}$ \\
\hline & Boron & SW846-6010B & 0.1 & $\mathrm{mg} / \mathrm{L}$ \\
\hline & Cadmium & EPA-200.8 & 0.0025 & $\mathrm{mg} / \mathrm{L}$ \\
\hline & Calcium & SW846-6010B & 0.2 & $\mathrm{mg} / \mathrm{L}$ \\
\hline & Chromium & EPA-200.8 & 0.01 & $\mathrm{mg} / \mathrm{L}$ \\
\hline & Cobalt & SW846-6010B & 0.02 & $\mathrm{mg} / \mathrm{L}$ \\
\hline & Copper & SW846-6010B & 0.02 & $\mathrm{mg} / \mathrm{L}$ \\
\hline & Iron & SW846-6010B & 0.05 & $\mathrm{mg} / \mathrm{L}$ \\
\hline & Lead & EPA-200.8 & 0.0005 & $\mathrm{mg} / \mathrm{L}$ \\
\hline & Lithium & SW846-6010B & 0.01 & $\mathrm{mg} / \mathrm{L}$ \\
\hline & Magnesium & SW846-6010B & 0.2 & $\mathrm{mg} / \mathrm{L}$ \\
\hline & Manganese & SW846-6010B & 0.005 & $\mathrm{mg} / \mathrm{L}$ \\
\hline & Mercury & SW846-7470 & 0.0002 & $\mathrm{mg} / \mathrm{L}$ \\
\hline & Molybdenum & SW846-6010B & 0.05 & $\mathrm{mg} / \mathrm{L}$ \\
\hline & Nickel & EPA-200.8 & 0.005 & $\mathrm{mg} / \mathrm{L}$ \\
\hline & Potassium & SW846-6010B & 2 & $\mathrm{mg} / \mathrm{L}$ \\
\hline & Selenium & EPA-200.8 & 0.01 & $\mathrm{mg} / \mathrm{L}$ \\
\hline & Silver & SW846-6010B & 0.02 & $\mathrm{mg} / \mathrm{L}$ \\
\hline
\end{tabular}


Table B.2 (continued)

\begin{tabular}{|c|c|c|c|c|}
\hline $\begin{array}{c}\text { Parameter } \\
\text { Group }\end{array}$ & Analyte & $\begin{array}{l}\text { Analytical } \\
\text { Method }^{1}\end{array}$ & $\begin{array}{c}\text { Reporting } \\
\text { Limit }^{2}\end{array}$ & Units $^{3}$ \\
\hline \multirow{7}{*}{$\begin{array}{c}\text { MET(1) } \\
\text { (continued) }\end{array}$} & Sodium & SW846-6010B & 0.2 & $\mathrm{mg} / \mathrm{L}$ \\
\hline & Strontium & SW846-6010B & 0.005 & $\mathrm{mg} / \mathrm{L}$ \\
\hline & Thallium & EPA-200.8 & 0.0005 & $\mathrm{mg} / \mathrm{L}$ \\
\hline & Thorium & SW846-6010B & 0.2 & $\mathrm{mg} / \mathrm{L}$ \\
\hline & Uranium & EPA-200.8 & 0.0005 & $\mathrm{mg} / \mathrm{L}$ \\
\hline & Vanadium & SW846-6010B & 0.02 & $\mathrm{mg} / \mathrm{L}$ \\
\hline & Zinc & SW846-6010B & 0.05 & $\mathrm{mg} / \mathrm{L}$ \\
\hline \multirow[t]{31}{*}{ VOC(1) } & Acetone & SW846-8260B-UP & 10 & $\mu \mathrm{g} / \mathrm{L}$ \\
\hline & Acrolein & SW846-8260B-UP & 10 & $\mu \mathrm{g} / \mathrm{L}$ \\
\hline & Acrylonitrile & SW846-8260B-UP & 5 & $\mu \mathrm{g} / \mathrm{L}$ \\
\hline & Benzene & SW846-8260B-UP & 5 & $\mu \mathrm{g} / \mathrm{L}$ \\
\hline & Bromochloromethane & SW846-8260B-UP & 5 & $\mu \mathrm{g} / \mathrm{L}$ \\
\hline & Bromodichloromethane & SW846-8260B-UP & 5 & $\mu \mathrm{g} / \mathrm{L}$ \\
\hline & Bromoform & SW846-8260B-UP & 5 & $\mu \mathrm{g} / \mathrm{L}$ \\
\hline & Bromomethane & SW846-8260B-UP & 5 & $\mu \mathrm{g} / \mathrm{L}$ \\
\hline & 2-Butanone & SW846-8260B-UP & 5 & $\mu \mathrm{g} / \mathrm{L}$ \\
\hline & Carbon disulfide & SW846-8260B-UP & 5 & $\mu \mathrm{g} / \mathrm{L}$ \\
\hline & Carbon tetrachloride & SW846-8260B-UP & 5 & $\mu \mathrm{g} / \mathrm{L}$ \\
\hline & Chlorobenzene & SW846-8260B-UP & 5 & $\mu \mathrm{g} / \mathrm{L}$ \\
\hline & Chloroethane & SW846-8260B-UP & 5 & $\mu \mathrm{g} / \mathrm{L}$ \\
\hline & 2-Chloroethylvinyl ether & SW846-8260B-UP & 10 & $\mu \mathrm{g} / \mathrm{L}$ \\
\hline & Chloroform & SW846-8260B-UP & 5 & $\mu \mathrm{g} / \mathrm{L}$ \\
\hline & Chloromethane & SW846-8260B-UP & 5 & $\mu \mathrm{g} / \mathrm{L}$ \\
\hline & Dibromochloromethane & SW846-8260B-UP & 5 & $\mu \mathrm{g} / \mathrm{L}$ \\
\hline & 1,2-Dibromo-3-chloropropane & SW846-8260B-UP & 10 & $\mu \mathrm{g} / \mathrm{L}$ \\
\hline & 1,2-Dibromoethane & SW846-8260B-UP & 5 & $\mu \mathrm{g} / \mathrm{L}$ \\
\hline & Dibromomethane & SW846-8260B-UP & 5 & $\mu \mathrm{g} / \mathrm{L}$ \\
\hline & 1,2-Dichlorobenzene & SW846-8260B-UP & 5 & $\mu \mathrm{g} / \mathrm{L}$ \\
\hline & 1,4-Dichlorobenzene & SW846-8260B-UP & 5 & $\mu \mathrm{g} / \mathrm{L}$ \\
\hline & 1,4-Dichloro-2-butene & SW846-8260B-UP & 5 & $\mu \mathrm{g} / \mathrm{L}$ \\
\hline & trans-1,4-Dichloro-2-butene & SW846-8260B-UP & 5 & $\mu \mathrm{g} / \mathrm{L}$ \\
\hline & Dichlorodifluoromethane & SW846-8260B-UP & 5 & $\mu \mathrm{g} / \mathrm{L}$ \\
\hline & 1,1-Dichloroethane & SW846-8260B-UP & 5 & $\mu \mathrm{g} / \mathrm{L}$ \\
\hline & 1,2-Dichloroethane & SW846-8260B-UP & 5 & $\mu \mathrm{g} / \mathrm{L}$ \\
\hline & 1,1-Dichloroethene & SW846-8260B-UP & 5 & $\mu \mathrm{g} / \mathrm{L}$ \\
\hline & cis-1,2-Dichloroethene & SW846-8260B-UP & 5 & $\mu \mathrm{g} / \mathrm{L}$ \\
\hline & trans-1,2-Dichloroethene & SW846-8260B-UP & 5 & $\mu \mathrm{g} / \mathrm{L}$ \\
\hline & 1,2-Dichloropropane & SW846-8260B-UP & 5 & $\mu \mathrm{g} / \mathrm{L}$ \\
\hline
\end{tabular}


Table B.2 (continued)

\begin{tabular}{|c|c|c|c|c|}
\hline $\begin{array}{c}\text { Parameter } \\
\text { Group }\end{array}$ & Analyte & $\begin{array}{c}\text { Analytical } \\
\text { Method }^{1} \\
\end{array}$ & $\begin{array}{c}\text { Reporting } \\
\text { Limit }^{2}\end{array}$ & Units $^{3}$ \\
\hline \multirow{23}{*}{$\begin{array}{c}\text { VOC(1) } \\
\text { (continued) }\end{array}$} & cis-1,3-Dichloropropene & SW846-8260B-UP & 5 & $\mu \mathrm{g} / \mathrm{L}$ \\
\hline & trans-1,3-Dichloropropene & SW846-8260B-UP & 5 & $\mu \mathrm{g} / \mathrm{L}$ \\
\hline & Ethanol & SW846-8260B-UP & 200 & $\mu \mathrm{g} / \mathrm{L}$ \\
\hline & Ethylbenzene & SW846-8260B-UP & 5 & $\mu \mathrm{g} / \mathrm{L}$ \\
\hline & Ethyl methacrylate & SW846-8260B-UP & 5 & $\mu \mathrm{g} / \mathrm{L}$ \\
\hline & 2-Hexanone & SW846-8260B-UP & 5 & $\mu \mathrm{g} / \mathrm{L}$ \\
\hline & Iodomethane & SW846-8260B-UP & 5 & $\mu \mathrm{g} / \mathrm{L}$ \\
\hline & 4-Methyl-2-pentanone & SW846-8260B-UP & 5 & $\mu \mathrm{g} / \mathrm{L}$ \\
\hline & Methylene chloride & SW846-8260B-UP & 5 & $\mu \mathrm{g} / \mathrm{L}$ \\
\hline & Styrene & SW846-8260B-UP & 5 & $\mu \mathrm{g} / \mathrm{L}$ \\
\hline & 1,1,1,2-Tetrachloroethane & SW846-8260B-UP & 5 & $\mu \mathrm{g} / \mathrm{L}$ \\
\hline & 1,1,2,2-Tetrachloroethane & SW846-8260B-UP & 5 & $\mu \mathrm{g} / \mathrm{L}$ \\
\hline & Tetrachloroethene & SW846-8260B-UP & 5 & $\mu \mathrm{g} / \mathrm{L}$ \\
\hline & Toluene & SW846-8260B-UP & 5 & $\mu \mathrm{g} / \mathrm{L}$ \\
\hline & Total Xylene & SW846-8260B-UP & 5 & $\mu \mathrm{g} / \mathrm{L}$ \\
\hline & 1,1,1-Trichloroethane & SW846-8260B-UP & 5 & $\mu \mathrm{g} / \mathrm{L}$ \\
\hline & 1,1,2-Trichloroethane & SW846-8260B-UP & 5 & $\mu \mathrm{g} / \mathrm{L}$ \\
\hline & Trichloroethene & SW846-8260B-UP & 5 & $\mu \mathrm{g} / \mathrm{L}$ \\
\hline & Trichlorofluoromethane & SW846-8260B-UP & 5 & $\mu \mathrm{g} / \mathrm{L}$ \\
\hline & 1,2,3-Trichloropropane & SW846-8260B-UP & 10 & $\mu \mathrm{g} / \mathrm{L}$ \\
\hline & 1,1,2-Trichloro-1,2,2-trifluoroethane & SW846-8260B-UP & 5 & $\mu \mathrm{g} / \mathrm{L}$ \\
\hline & Vinyl acetate & SW846-8260B-UP & 10 & $\mu \mathrm{g} / \mathrm{L}$ \\
\hline & Vinyl chloride & SW846-8260B-UP & 2 & $\mu \mathrm{g} / \mathrm{L}$ \\
\hline $\mathrm{RAD}(1)$ & Gross Alpha Activity & EPA-900.0 & 3.5 & $\mathrm{pCi} / \mathrm{L}$ \\
\hline RAD(1) & Gross Beta Activity & EPA-900.0 & 7.0 & $\mathrm{pCi} / \mathrm{L}$ \\
\hline RAD(3) & Uranium-234, $-235, \&-238$ & Y/P65-7061 & 0.4 & $\mathrm{pCi} / \mathrm{L}$ \\
\hline $\mathrm{RAD}(12)$ & Technetium-99 & Y/P65-7060 & 10 & $\mathrm{pCi} / \mathrm{L}$ \\
\hline RAD(13) & Total Uranium and weight \% U-235 & Y/P65-8044 & 0.002 & $\mathrm{mg} / \mathrm{L}$ \\
\hline
\end{tabular}

\section{Notes:}

$1 \quad$ NA - not applicable

Field measurement procedures:

- $\quad$ BWXT 2000a, BWXT 2002a, BWXT 2004a, and BWXT 2004b

Analytical methods from:

Test Methods for Evaluating Solid Waste Physical/Chemical Methods

(U.S. Environmental Protection Agency 1996)

Methods for Chemical Analysis of Water and Wastes

(U.S. Environmental Protection Agency 1983)

- BWXT Analytical Chemistry Organization Controlled Procedures:

(Y/P65-7060 and Y/P65-7061) 


\section{Table B.2 (continued)}

Notes: (continued)

2

NA - not applicable

VOC(1) - Reporting limits are contract-required quantitation limits; also report estimated values (with qualifier) below this limit and above the instrument detection limit.

RAD(1,3,12) - Reporting limits are target minimum detectable activities (MDAs) that may be obtained under optimal analytical conditions; actual MDAs are samplespecific and may vary significantly from the target value.

3

$\mathrm{ft}$ - feet

$\mu \mathrm{g} / \mathrm{L} \quad$ - micrograms per liter

$\mu \mathrm{mho} / \mathrm{cm}$ - micromhos per centimeter

$\mathrm{mg} / \mathrm{L}$ - milligrams per liter

$\mathrm{mV}$ - millivolts

NTU - nephelometric turbidity units

ppm - parts per million

pCi/L - picoCuries per liter 
APPENDIX C

SAMPLING PRIORITY SCORES 


\section{Sampling Priority Scores for CY 2005}

\begin{tabular}{|c|c|c|c|c|c|c|c|c|c|c|}
\hline \multirow[b]{2}{*}{ Rank } & \multirow{2}{*}{$\begin{array}{c}\text { Sampling } \\
\text { Priority } \\
\text { Score }^{1}\end{array}$} & \multirow[b]{2}{*}{ Well No. } & \multirow{2}{*}{$\begin{array}{c}\text { Hydro. } \\
\text { Regime }^{2}\end{array}$} & \multirow{2}{*}{$\begin{array}{c}\text { Most } \\
\text { Recent } \\
\text { Sample }^{3}\end{array}$} & \multicolumn{5}{|c|}{ Points Scored $^{4}$} & \multirow{2}{*}{$\begin{array}{l}\text { Selected for } \\
\text { CY } 2005^{5}\end{array}$} \\
\hline & & & & & Regime & $\begin{array}{c}\text { Time since } \\
\text { sampled last }\end{array}$ & $\begin{array}{c}\text { No. of } \\
\text { Samples }\end{array}$ & $\begin{array}{c}\text { Contaminant } \\
\text { Concentrations }\end{array}$ & $\begin{array}{l}\text { Trending } \\
\text { Potential }\end{array}$ & \\
\hline 1 & 19 & GW-243 & $\mathrm{BC}$ & $08 / 13 / 02$ & 5 & 1 & -5 & 8 & 10 & \\
\hline 2 & 19 & GW-246 & $\mathrm{BC}$ & 08/15/04 & 5 & 0 & -5 & 9 & 10 & \\
\hline 3 & 17 & GW-052 & $\mathrm{BC}$ & 08/15/04 & 5 & 0 & -1 & 3 & 10 & \\
\hline 4 & 16 & GW-097 & BC & 07/15/98 & 5 & 5 & -5 & 1 & 10 & $\mathrm{x}$ \\
\hline 5 & 16 & GW-624 & BC & 07/15/98 & 5 & 5 & -2 & 1 & 7 & $x$ \\
\hline 6 & 16 & GW-242 & BC & 03/08/99 & 5 & 4 & -5 & 2 & 10 & $x$ \\
\hline 7 & 16 & GW-346 & BC & 03/09/99 & 5 & 4 & -4 & 2 & 9 & $\mathrm{x}$ \\
\hline 8 & 16 & GW-228 & BC & 09/09/99 & 5 & 4 & -5 & 2 & 10 & $x$ \\
\hline 9 & 15 & GW-064 & BC & 07/20/98 & 5 & 5 & -5 & 1 & 9 & $x$ \\
\hline 10 & 15 & GW-601 & BC & 03/08/99 & 5 & 4 & -5 & 3 & 8 & $x$ \\
\hline 11 & 15 & GW-066 & BC & 08/13/02 & 5 & 1 & 3 & 1 & 5 & $x$ \\
\hline 12 & 15 & GW-101 & $\mathrm{BC}$ & 08/15/04 & 5 & 0 & -3 & 3 & 10 & \\
\hline 13 & 15 & GW-257 & $\mathrm{BC}$ & 08/15/04 & 5 & 0 & -2 & 2 & 10 & \\
\hline 14 & 15 & GW-615 & $\mathrm{BC}$ & 08/15/04 & 5 & 0 & -5 & 5 & 10 & \\
\hline 15 & 14 & GW-227 & BC & 09/12/92 & 5 & 5 & -5 & 4 & 5 & $\mathrm{x}$ \\
\hline 16 & 14 & GW-365 & BC & $08 / 07 / 01$ & 5 & 2 & -5 & 2 & 10 & $x$ \\
\hline 17 & 14 & GW-124 & BC & 08/09/01 & 5 & 2 & -5 & 2 & 10 & $x$ \\
\hline 18 & 14 & GW-616 & BC & 08/09/01 & 5 & 2 & -5 & 2 & 10 & $x$ \\
\hline 19 & 14 & GW-061 & BC & 08/05/02 & 5 & 1 & -5 & 3 & 10 & $x$ \\
\hline 20 & 13 & GW-312 & BC & $08 / 06 / 95$ & 5 & 5 & -5 & 2 & 6 & $x$ \\
\hline 21 & 13 & GW-053 & BC & $07 / 25 / 01$ & 5 & 2 & -5 & 1 & 10 & $x$ \\
\hline 22 & 13 & GW-364 & BC & 08/07/01 & 5 & 2 & -5 & 1 & 10 & $x$ \\
\hline 23 & 13 & GW-626 & BC & $07 / 25 / 02$ & 5 & 1 & -5 & 2 & 10 & $x$ \\
\hline 24 & 13 & GW-072 & BC & 08/06/02 & 5 & 1 & -5 & 2 & 10 & $x$ \\
\hline 25 & 13 & GW-288 & BC & $08 / 07 / 02$ & 5 & 1 & -5 & 2 & 10 & $x$ \\
\hline 26 & 13 & GW-291 & BC & $08 / 07 / 02$ & 5 & 1 & -5 & 2 & 10 & $x$ \\
\hline 27 & 13 & GW-289 & BC & $08 / 08 / 02$ & 5 & 1 & -5 & 2 & 10 & $x$ \\
\hline 28 & 13 & GW-014 & BC & 08/12/02 & 5 & 1 & -5 & 2 & 10 & $x$ \\
\hline 29 & 12 & GW-245 & BC & $01 / 17 / 90$ & 5 & 5 & -5 & 6 & 1 & $x$ \\
\hline 30 & 12 & GW-127 & BC & 01/18/90 & 5 & 5 & -5 & 5 & 2 & $x$ \\
\hline 31 & 12 & GW-277 & BC & 01/23/90 & 5 & 5 & -5 & 5 & 2 & $x$ \\
\hline 32 & 12 & GW-694 & BC & $07 / 17 / 02$ & 5 & 1 & -5 & 1 & 10 & $x$ \\
\hline 33 & 12 & GW-736 & BC & $07 / 17 / 02$ & 5 & 1 & -5 & 2 & 9 & $x$ \\
\hline 34 & 12 & GW-737 & BC & 07/18/02 & 5 & 1 & -5 & 2 & 9 & $x$ \\
\hline 35 & 12 & GW-100 & BC & 08/15/04 & 5 & 0 & -5 & 2 & 10 & \\
\hline 36 & 12 & GW-236 & $\mathrm{BC}$ & 08/15/04 & 5 & 0 & -5 & 2 & 10 & \\
\hline 37 & 11 & GW-247 & BC & $01 / 17 / 90$ & 5 & 5 & -5 & 5 & 1 & $x$ \\
\hline 38 & 11 & GW-244 & BC & 01/18/90 & 5 & 5 & -5 & 5 & 1 & $x$ \\
\hline 39 & 11 & GW-368 & BC & $01 / 31 / 90$ & 5 & 5 & -2 & 2 & 1 & $x$ \\
\hline 40 & 11 & GW-068 & BC & $03 / 12 / 90$ & 5 & 5 & -3 & 2 & 2 & $x$ \\
\hline 41 & 11 & GW-723 & BC & $07 / 23 / 02$ & 5 & 1 & -5 & 1 & 9 & $x$ \\
\hline 42 & 11 & GW-739 & BC & 07/22/02 & 5 & 1 & -5 & 1 & 9 & $x$ \\
\hline 43 & 11 & GW-710 & $\mathrm{BC}$ & 07/14/03 & 5 & 0 & -5 & 1 & 10 & \\
\hline 44 & 10 & GW-259 & $\mathrm{BC}$ & $03 / 05 / 90$ & 5 & 5 & -3 & 2 & 1 & \\
\hline 45 & 10 & GW-122 & BC & 04/13/91 & 5 & 5 & -5 & 2 & 3 & $x$ \\
\hline 46 & 10 & GW-307 & BC & $04 / 26 / 91$ & 5 & 5 & -5 & 3 & 2 & $x$ \\
\hline 47 & 10 & GW-645 & BC & $08 / 02 / 92$ & 5 & 5 & -2 & 1 & 1 & $x$ \\
\hline 48 & 10 & GW-314 & $\mathrm{BC}$ & 08/15/92 & 5 & 5 & -5 & 2 & 3 & \\
\hline 49 & 10 & GW-309 & $\mathrm{BC}$ & 08/30/92 & 5 & 5 & -5 & 2 & 3 & \\
\hline 50 & 10 & GW-835 & $\mathrm{BC}$ & $08 / 21 / 03$ & 5 & 0 & -5 & 5 & 5 & \\
\hline 51 & 9 & GW-258 & $\mathrm{BC}$ & $03 / 05 / 90$ & 5 & 5 & -3 & 1 & 1 & \\
\hline 52 & 9 & GW-306 & $\mathrm{BC}$ & 04/26/91 & 5 & 5 & -5 & 2 & 2 & \\
\hline 53 & 9 & GW-308 & $\mathrm{BC}$ & 04/30/91 & 5 & 5 & -5 & 2 & 2 & \\
\hline 54 & 9 & GW-310 & BC & 04/30/91 & 5 & 5 & -5 & 2 & 2 & $x$ \\
\hline 55 & 9 & GW-018 & $\mathrm{BC}$ & 06/20/91 & 5 & 5 & 4 & 0 & -5 & \\
\hline
\end{tabular}


Sampling Priority Scores for CY 2005

\begin{tabular}{|c|c|c|c|c|c|c|c|c|c|c|}
\hline \multirow[b]{2}{*}{ Rank } & \multirow{2}{*}{\begin{tabular}{|c|} 
Sampling \\
Priority \\
Score $^{1}$
\end{tabular}} & \multirow[b]{2}{*}{ Well No. } & \multirow{2}{*}{$\begin{array}{c}\text { Hydro. } \\
\text { Regime }^{2}\end{array}$} & \multirow{2}{*}{$\begin{array}{c}\text { Most } \\
\text { Recent } \\
\text { Sample }^{3}\end{array}$} & \multicolumn{5}{|c|}{ Points Scored ${ }^{4}$} & \multirow{2}{*}{$\begin{array}{l}\text { Selected for } \\
\text { CY } 2005^{5}\end{array}$} \\
\hline & & & & & Regime & $\begin{array}{l}\text { Time since } \\
\text { sampled last }\end{array}$ & $\begin{array}{c}\text { No. of } \\
\text { Samples }\end{array}$ & $\begin{array}{c}\text { Contaminant } \\
\text { Concentrations }\end{array}$ & $\begin{array}{l}\text { Trending } \\
\text { Potential }\end{array}$ & \\
\hline 56 & 9 & GW-313 & BC & $08 / 15 / 92$ & 5 & 5 & -5 & 1 & 3 & $x$ \\
\hline 57 & 9 & GW-623 & $\mathrm{BC}$ & 09/27/92 & 5 & 5 & -3 & 1 & 1 & \\
\hline 58 & 8 & GW-010 & $\mathrm{BC}$ & $02 / 01 / 90$ & 5 & 5 & -5 & 2 & 1 & \\
\hline 59 & 8 & GW-369 & $\mathrm{BC}$ & 05/15/91 & 5 & 5 & -5 & 2 & 1 & \\
\hline 60 & 8 & GW-829 & $\mathrm{BC}$ & 08/01/01 & 5 & 2 & -5 & 1 & 5 & \\
\hline 61 & 8 & $56-3 C$ & EF & $03 / 12 / 97$ & -5 & 5 & 5 & 2 & 1 & \\
\hline 62 & 8 & $55-2 B$ & EF & $11 / 20 / 04$ & -5 & 0 & 2 & 4 & 7 & \\
\hline 63 & 7 & GW-367 & $\mathrm{BC}$ & 05/11/91 & 5 & 5 & -5 & 1 & 1 & \\
\hline 64 & 7 & GW-089 & $\mathrm{BC}$ & 08/13/92 & 5 & 5 & -5 & 1 & 1 & \\
\hline 65 & 7 & GW-006 & $\mathrm{BC}$ & $08 / 02 / 00$ & 5 & 3 & -3 & 1 & 1 & \\
\hline 66 & 6 & GW-249 & $\mathrm{BC}$ & $02 / 16 / 90$ & 5 & 5 & 1 & 0 & -5 & \\
\hline 67 & 6 & GW-274 & EF & $10 / 22 / 03$ & -5 & 0 & -5 & 6 & 10 & \\
\hline 68 & 6 & GW-692 & EF & $11 / 20 / 04$ & -5 & 0 & 3 & 1 & 7 & \\
\hline 69 & 5 & GW-091 & $\mathrm{BC}$ & 08/08/02 & 5 & 1 & 4 & 0 & -5 & \\
\hline 70 & 5 & GW-237 & $\mathrm{BC}$ & 08/15/04 & 5 & 0 & 4 & 1 & -5 & \\
\hline 71 & 5 & GW-265 & EF & 03/13/97 & -5 & 5 & -5 & 1 & 9 & \\
\hline 72 & 5 & GW-332 & EF & 08/04/98 & -5 & 5 & -5 & 2 & 8 & \\
\hline 73 & 5 & GW-148 & EF & $11 / 09 / 99$ & -5 & 4 & -5 & 1 & 10 & \\
\hline 74 & 5 & GW-109 & EF & $10 / 06 / 03$ & -5 & 0 & -5 & 5 & 10 & \\
\hline 75 & 4 & GW-045 & $\mathrm{BC}$ & $02 / 08 / 90$ & 5 & 5 & -1 & 0 & -5 & \\
\hline 76 & 4 & GW-275 & EF & $10 / 22 / 03$ & -5 & 0 & -5 & 4 & 10 & \\
\hline 77 & 4 & GW-700 & EF & $11 / 10 / 03$ & -5 & 0 & 1 & 2 & 6 & \\
\hline 78 & 4 & GW-690 & EF & $11 / 18 / 03$ & -5 & 0 & 1 & 2 & 6 & \\
\hline 79 & 4 & GW-222 & EF & $11 / 20 / 04$ & -5 & 0 & -5 & 4 & 10 & \\
\hline 80 & 3 & GW-375 & $\mathrm{BC}$ & $02 / 06 / 90$ & 5 & 5 & -2 & 0 & -5 & \\
\hline 81 & 3 & GW-648 & $\mathrm{BC}$ & $07 / 23 / 92$ & 5 & 5 & -2 & 0 & -5 & \\
\hline 82 & 3 & GW-646 & $\mathrm{BC}$ & $08 / 03 / 92$ & 5 & 5 & -2 & 0 & -5 & \\
\hline 83 & 3 & GW-619 & $\mathrm{EF}$ & $07 / 21 / 98$ & -5 & 5 & -5 & 1 & 7 & \\
\hline 84 & 3 & GW-659 & EF & $07 / 28 / 98$ & -5 & 5 & -5 & 2 & 6 & \\
\hline 85 & 3 & GW-180 & $\mathrm{CR}$ & $08 / 21 / 01$ & -5 & 2 & -5 & 1 & 10 & \\
\hline 86 & 3 & GW-192 & EF & $10 / 17 / 01$ & -5 & 2 & -5 & 1 & 10 & \\
\hline 87 & 3 & GW-240 & EF & $10 / 22 / 01$ & -5 & 2 & -5 & 1 & 10 & \\
\hline 88 & 3 & GW-656 & EF & $11 / 12 / 01$ & -5 & 2 & -5 & 2 & 9 & \\
\hline 89 & 2 & GW-183 & EF & 03/18/97 & -5 & 5 & -5 & 2 & 5 & \\
\hline 90 & 2 & GW-617 & EF & $11 / 05 / 97$ & -5 & 5 & -5 & 1 & 6 & \\
\hline 91 & 2 & GW-105 & EF & $10 / 02 / 03$ & -5 & 0 & -5 & 2 & 10 & \\
\hline 92 & 2 & GW-106 & EF & $10 / 02 / 03$ & -5 & 0 & -5 & 2 & 10 & \\
\hline 93 & 2 & GW-270 & EF & $10 / 20 / 03$ & -5 & 0 & -5 & 2 & 10 & \\
\hline 94 & 2 & GW-269 & EF & $10 / 23 / 03$ & -5 & 0 & -5 & 2 & 10 & \\
\hline 95 & 2 & GW-272 & EF & $10 / 23 / 03$ & -5 & 0 & -5 & 2 & 10 & \\
\hline 96 & 2 & GW-336 & EF & $11 / 17 / 03$ & -5 & 0 & -5 & 2 & 10 & \\
\hline 97 & 2 & GW-176 & $\mathrm{CR}$ & $11 / 20 / 04$ & -5 & 0 & -5 & 2 & 10 & \\
\hline 98 & 2 & GW-179 & CR & $11 / 20 / 04$ & -5 & 0 & -5 & 2 & 10 & \\
\hline 99 & 2 & GW-322 & CR & $11 / 20 / 04$ & -5 & 0 & -5 & 2 & 10 & \\
\hline 100 & 2 & GW-612 & CR & $11 / 20 / 04$ & -5 & 0 & -5 & 2 & 10 & \\
\hline 101 & 1 & GW-531 & $\mathrm{BC}$ & $06 / 24 / 91$ & 5 & 5 & -4 & 0 & -5 & \\
\hline 102 & 1 & GW-086 & $\mathrm{BC}$ & $10 / 21 / 93$ & 5 & 5 & -4 & 0 & -5 & \\
\hline 103 & 1 & GW-248 & $\mathrm{BC}$ & $08 / 08 / 95$ & 5 & 5 & -4 & 0 & -5 & \\
\hline 104 & 1 & GW-629 & $\mathrm{BC}$ & $07 / 16 / 98$ & 5 & 5 & -4 & 0 & -5 & \\
\hline 105 & 1 & GW-505 & EF & $10 / 06 / 03$ & -5 & 0 & -5 & 1 & 10 & \\
\hline 106 & 1 & GW-273 & EF & $10 / 21 / 03$ & -5 & 0 & -5 & 1 & 10 & \\
\hline 107 & 1 & GW-337 & EF & $11 / 17 / 03$ & -5 & 0 & -5 & 1 & 10 & \\
\hline 108 & 1 & $56-2 C$ & EF & $11 / 18 / 03$ & -5 & 0 & -1 & 2 & 5 & \\
\hline 109 & 1 & GW-173 & CR & $11 / 20 / 04$ & -5 & 0 & -5 & 1 & 10 & \\
\hline 110 & 1 & GW-175 & $\mathrm{CR}$ & $11 / 20 / 04$ & -5 & 0 & -5 & 1 & 10 & \\
\hline
\end{tabular}




\section{Sampling Priority Scores for CY 2005}

\begin{tabular}{|c|c|c|c|c|c|c|c|c|c|c|}
\hline \multirow[b]{2}{*}{ Rank } & \multirow{2}{*}{$\begin{array}{c}\text { Sampling } \\
\text { Priority } \\
\text { Score }^{1}\end{array}$} & \multirow[b]{2}{*}{ Well No. } & \multirow[b]{2}{*}{$\begin{array}{c}\text { Hydro. } \\
\text { Regime }^{2}\end{array}$} & \multirow{2}{*}{$\begin{array}{c}\text { Most } \\
\text { Recent }^{2} \\
\text { Sample }^{3}\end{array}$} & \multicolumn{5}{|c|}{ Points Scored ${ }^{4}$} & \multirow{2}{*}{$\begin{array}{l}\text { Selected for } \\
\text { CY } 2005^{5}\end{array}$} \\
\hline & & & & & Regime & $\begin{array}{c}\text { Time since } \\
\text { sampled last }\end{array}$ & $\begin{array}{c}\text { No. of } \\
\text { Samples }\end{array}$ & $\begin{array}{c}\text { Contaminant } \\
\text { Concentrations }\end{array}$ & $\begin{array}{l}\text { Trending } \\
\text { Potential }\end{array}$ & \\
\hline 111 & 1 & GW-178 & $\mathrm{CR}$ & $11 / 20 / 04$ & -5 & 0 & -5 & 1 & 10 & \\
\hline 112 & 0 & GW-073 & $\mathrm{BC}$ & $01 / 30 / 90$ & 5 & 5 & -5 & 0 & -5 & \\
\hline 113 & 0 & GW-120 & $\mathrm{BC}$ & $01 / 30 / 90$ & 5 & 5 & -5 & 0 & -5 & \\
\hline 114 & 0 & GW-094 & $\mathrm{BC}$ & $02 / 15 / 90$ & 5 & 5 & -5 & 0 & -5 & \\
\hline 115 & 0 & GW-250 & $\mathrm{BC}$ & $02 / 16 / 90$ & 5 & 5 & -5 & 0 & -5 & \\
\hline 116 & 0 & GW-067 & $\mathrm{BC}$ & 03/13/90 & 5 & 5 & -5 & 0 & -5 & \\
\hline 117 & 0 & GW-074 & $\mathrm{BC}$ & 08/03/92 & 5 & 5 & -5 & 0 & -5 & \\
\hline 118 & 0 & GW-630 & $\mathrm{BC}$ & 08/29/92 & 5 & 5 & -5 & 0 & -5 & \\
\hline 119 & 0 & GW-054 & $\mathrm{BC}$ & 09/02/92 & 5 & 5 & -5 & 0 & -5 & \\
\hline 120 & 0 & GW-083 & $\mathrm{BC}$ & 09/02/92 & 5 & 5 & -5 & 0 & -5 & \\
\hline 121 & 0 & GW-366 & BC & 09/07/92 & 5 & 5 & -5 & 0 & -5 & \\
\hline 122 & 0 & GW-520 & $\mathrm{BC}$ & 09/07/92 & 5 & 5 & -5 & 0 & -5 & \\
\hline 123 & 0 & GW-075 & BC & 09/08/92 & 5 & 5 & -5 & 0 & -5 & \\
\hline 124 & 0 & GW-125 & $\mathrm{BC}$ & 09/17/92 & 5 & 5 & -5 & 0 & -5 & \\
\hline 125 & 0 & GW-058 & $\mathrm{BC}$ & 09/24/92 & 5 & 5 & -5 & 0 & -5 & \\
\hline 126 & 0 & GW-622 & $\mathrm{BC}$ & 09/25/92 & 5 & 5 & -5 & 0 & -5 & \\
\hline 127 & 0 & GW-286 & BC & $10 / 07 / 93$ & 5 & 5 & -5 & 0 & -5 & $x$ \\
\hline 128 & 0 & GW-651 & BC & 10/10/93 & 5 & 5 & -5 & 0 & -5 & \\
\hline 129 & 0 & GW-641 & $\mathrm{BC}$ & $10 / 14 / 93$ & 5 & 5 & -5 & 0 & -5 & \\
\hline 130 & 0 & GW-316 & $\mathrm{BC}$ & $10 / 24 / 93$ & 5 & 5 & -5 & 0 & -5 & \\
\hline 131 & 0 & GW-323 & $\mathrm{BC}$ & $10 / 23 / 93$ & 5 & 5 & -5 & 0 & -5 & \\
\hline 132 & 0 & GW-636 & BC & $12 / 18 / 93$ & 5 & 5 & -5 & 0 & -5 & \\
\hline 133 & 0 & GW-638 & $\mathrm{BC}$ & $12 / 18 / 93$ & 5 & 5 & -5 & 0 & -5 & \\
\hline 134 & 0 & GW-013 & BC & $12 / 19 / 93$ & 5 & 5 & -5 & 0 & -5 & \\
\hline 135 & 0 & GW-325 & $\mathrm{BC}$ & $02 / 15 / 94$ & 5 & 5 & -5 & 0 & -5 & \\
\hline 136 & 0 & GW-637 & $\mathrm{BC}$ & 07/19/95 & 5 & 5 & -5 & 0 & -5 & \\
\hline 137 & 0 & GW-047 & $B C$ & 07/24/95 & 5 & 5 & -5 & 0 & -5 & \\
\hline 138 & 0 & GW-057 & $\mathrm{BC}$ & 08/05/95 & 5 & 5 & -5 & 0 & -5 & \\
\hline 139 & 0 & GW-800 & $\mathrm{BC}$ & 08/05/95 & 5 & 5 & -5 & 0 & -5 & \\
\hline 140 & 0 & GW-290 & $\mathrm{BC}$ & 08/07/95 & 5 & 5 & -5 & 0 & -5 & \\
\hline 141 & 0 & GW-370 & BC & 08/08/95 & 5 & 5 & -5 & 0 & -5 & \\
\hline 142 & 0 & GW-317 & $\mathrm{BC}$ & 08/15/95 & 5 & 5 & -5 & 0 & -5 & \\
\hline 143 & 0 & GW-347 & $\mathrm{BC}$ & 08/15/95 & 5 & 5 & -5 & 0 & -5 & \\
\hline 144 & 0 & GW-348 & $\mathrm{BC}$ & 08/21/95 & 5 & 5 & -5 & 0 & -5 & \\
\hline 145 & 0 & GW-652 & BC & 09/19/95 & 5 & 5 & -5 & 0 & -5 & \\
\hline 146 & 0 & GW-654 & $\mathrm{BC}$ & $12 / 09 / 95$ & 5 & 5 & -5 & 0 & -5 & \\
\hline 147 & 0 & GW-794 & $\mathrm{BC}$ & 08/26/96 & 5 & 5 & -5 & 0 & -5 & \\
\hline 148 & 0 & GW-795 & $\mathrm{BC}$ & 08/26/96 & 5 & 5 & -5 & 0 & -5 & \\
\hline 149 & 0 & GW-095 & BC & $08 / 27 / 96$ & 5 & 5 & -5 & 0 & -5 & \\
\hline 150 & 0 & GW-613 & $\mathrm{BC}$ & 08/11/97 & 5 & 5 & -5 & 0 & -5 & \\
\hline 151 & 0 & GW-084 & $\mathrm{BC}$ & 08/13/97 & 5 & 5 & -5 & 0 & -5 & \\
\hline 152 & 0 & GW-372 & BC & $08 / 14 / 97$ & 5 & 5 & -5 & 0 & -5 & \\
\hline 153 & 0 & GW-642 & $\mathrm{BC}$ & $08 / 14 / 97$ & 5 & 5 & -5 & 0 & -5 & \\
\hline 154 & 0 & GW-001 & $\mathrm{BC}$ & 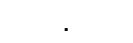 & 5 & . & & . & -5 & \\
\hline 155 & 0 & GW-012 & $B C$ & . & 5 & . & . & . & -5 & \\
\hline 156 & 0 & GW-016 & BC & . & 5 & . & . & . & -5 & \\
\hline 157 & 0 & GW-041 & $B C$ & . & 5 & . & . & . & -5 & \\
\hline 158 & 0 & GW-055 & $B C$ & . & 5 & . & . & . & -5 & \\
\hline 159 & 0 & GW-059 & BC & . & 5 & . & 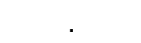 & . & -5 & \\
\hline 160 & 0 & GW-065 & $\mathrm{BC}$ & . & 5 & . & . & . & -5 & \\
\hline 161 & 0 & GW-090 & $\mathrm{BC}$ & & 5 & & & . & -5 & \\
\hline 162 & 0 & $55-1 C$ & EF & $06 / 07 / 96$ & -5 & 5 & 5 & 0 & -5 & \\
\hline 163 & 0 & $56-1 C$ & EF & 03/13/97 & -5 & 5 & 5 & 0 & -5 & \\
\hline 164 & 0 & GW-699 & EF & 03/12/97 & -5 & 5 & 5 & 0 & -5 & \\
\hline 165 & 0 & GW-819 & EF & 03/26/98 & -5 & 5 & 5 & 0 & -5 & \\
\hline
\end{tabular}




\section{Sampling Priority Scores for CY 2005}

\begin{tabular}{|c|c|c|c|c|c|c|c|c|c|c|}
\hline \multirow[b]{2}{*}{ Rank } & \multirow{2}{*}{$\begin{array}{c}\text { Sampling } \\
\text { Priority } \\
\text { Score }^{1} \\
\end{array}$} & \multirow[b]{2}{*}{ Well No. } & \multirow[b]{2}{*}{$\begin{array}{c}\text { Hydro. } \\
\text { Regime }^{2}\end{array}$} & \multirow{2}{*}{$\begin{array}{c}\text { Most } \\
\text { Recent } \\
\text { Sample }^{3} \\
\end{array}$} & \multicolumn{5}{|c|}{ Points Scored ${ }^{4}$} & \multirow{2}{*}{$\begin{array}{l}\text { Selected for } \\
\text { CY } 2005^{5}\end{array}$} \\
\hline & & & & & Regime & $\begin{array}{c}\text { Time since } \\
\text { sampled last }\end{array}$ & $\begin{array}{c}\text { No. of } \\
\text { Samples }\end{array}$ & $\begin{array}{c}\text { Contaminant } \\
\text { Concentrations }\end{array}$ & $\begin{array}{l}\text { Trending } \\
\text { Potential } \\
\end{array}$ & \\
\hline 166 & 0 & GW-775 & $\mathrm{EF}$ & $10 / 31 / 02$ & -5 & 1 & -5 & 1 & 8 & \\
\hline 167 & 0 & $55-2 C$ & $\mathrm{EF}$ & 11/18/03 & -5 & 0 & -5 & 4 & 6 & \\
\hline 168 & -1 & GW-126 & $B C$ & 03/02/99 & 5 & 4 & -5 & 0 & -5 & \\
\hline 169 & -1 & GW-345 & $B C$ & 03/09/99 & 5 & 4 & -5 & 0 & -5 & \\
\hline 170 & -1 & GW-334 & $\mathrm{EF}$ & 04/13/91 & -5 & 5 & -4 & 2 & 1 & \\
\hline 171 & -1 & $55-3 C$ & $\mathrm{EF}$ & $04 / 24 / 97$ & -5 & 5 & 4 & 0 & -5 & \\
\hline 172 & -1 & GW-783 & $\mathrm{EF}$ & 05/01/00 & -5 & 3 & -5 & 2 & 4 & \\
\hline 173 & -1 & GW-820 & $\mathrm{EF}$ & 10/13/03 & -5 & 0 & -1 & 1 & 4 & \\
\hline 174 & -2 & GW-621 & $\mathrm{BC}$ & 07/13/00 & 5 & 3 & -5 & 0 & -5 & \\
\hline 175 & -2 & GW-287 & BC & $08 / 21 / 00$ & 5 & 3 & -5 & 0 & -5 & $x$ \\
\hline 176 & -2 & GW-107 & $\mathrm{EF}$ & 01/19/90 & -5 & 5 & -5 & 1 & 2 & \\
\hline 177 & -2 & GW-282 & $\mathrm{EF}$ & 07/29/92 & -5 & 5 & -5 & 1 & 2 & \\
\hline 178 & -2 & GW-508 & $\mathrm{EF}$ & 03/02/94 & -5 & 5 & -5 & 1 & 2 & \\
\hline 179 & -3 & GW-056 & $B C$ & 03/14/01 & 5 & 2 & -5 & 0 & -5 & \\
\hline 180 & -3 & GW-685 & $B C$ & 03/14/01 & 5 & 2 & -5 & 0 & -5 & \\
\hline 181 & -4 & GW-069 & $B C$ & 08/05/02 & 5 & 1 & -5 & 0 & -5 & \\
\hline 182 & -4 & GW-778 & $\mathrm{EF}$ & 05/18/95 & -5 & 5 & 1 & 0 & -5 & \\
\hline 183 & -4 & GW-686 & $\mathrm{EF}$ & $11 / 14 / 02$ & -5 & 1 & 4 & 1 & -5 & \\
\hline 184 & -4 & $56-2 A$ & $\mathrm{EF}$ & $11 / 20 / 04$ & -5 & 0 & 4 & 2 & -5 & \\
\hline 185 & -4 & $56-2 B$ & $\mathrm{EF}$ & $11 / 20 / 04$ & -5 & 0 & 4 & 2 & -5 & \\
\hline 186 & -4 & GW-691 & $\mathrm{EF}$ & $11 / 20 / 04$ & -5 & 0 & 4 & 2 & -5 & \\
\hline 187 & -5 & GW-711 & $B C$ & 07/14/03 & 5 & 0 & -5 & 0 & -5 & \\
\hline 188 & -5 & GW-123 & $B C$ & 08/04/03 & 5 & 0 & -5 & 0 & -5 & \\
\hline 189 & -5 & 55-1B & $\mathrm{EF}$ & $10 / 14 / 02$ & -5 & 1 & 4 & 0 & -5 & \\
\hline 190 & -5 & $55-1 \mathrm{~A}$ & $\mathrm{EF}$ & $11 / 20 / 04$ & -5 & 0 & 4 & 1 & -5 & \\
\hline 191 & -5 & GW-679 & $\mathrm{CR}$ & $11 / 20 / 04$ & -5 & 0 & 5 & . & -5 & \\
\hline 192 & -5 & GW-680 & $\mathrm{CR}$ & $11 / 20 / 04$ & -5 & 0 & 5 & . & -5 & \\
\hline 193 & -5 & GW-818 & $\mathrm{EF}$ & $11 / 20 / 04$ & -5 & 0 & 4 & 1 & -5 & \\
\hline 194 & -6 & $60-1 B$ & $\mathrm{EF}$ & 10/13/03 & -5 & 0 & 4 & 0 & -5 & \\
\hline 195 & -6 & $55-6 \mathrm{~A}$ & $\mathrm{EF}$ & $11 / 20 / 04$ & -5 & 0 & 4 & 0 & -5 & \\
\hline 196 & -7 & GW-167 & $\mathrm{EF}$ & 02/14/96 & -5 & 5 & -2 & 0 & -5 & \\
\hline 197 & -7 & $59-1 \mathrm{~A}$ & $\mathrm{EF}$ & $10 / 30 / 03$ & -5 & 0 & 3 & 0 & -5 & \\
\hline 198 & -8 & GW-200 & $\mathrm{EF}$ & 10/10/88 & -5 & 5 & -3 & 0 & -5 & \\
\hline 199 & -8 & GW-202 & $\mathrm{EF}$ & 10/10/88 & -5 & 5 & -3 & 0 & -5 & \\
\hline 200 & -8 & GW-218 & $\mathrm{EF}$ & $11 / 06 / 00$ & -5 & 3 & -1 & 0 & -5 & \\
\hline 201 & -8 & $59-1 C$ & $\mathrm{EF}$ & 10/30/03 & -5 & 0 & 2 & 0 & -5 & \\
\hline 202 & -8 & $59-1 B$ & $E F$ & $11 / 20 / 04$ & -5 & 0 & 2 & 0 & -5 & \\
\hline 203 & -9 & GW-150 & $\mathrm{EF}$ & 03/08/88 & -5 & 5 & -4 & 0 & -5 & \\
\hline 204 & -9 & GW-335 & $\mathrm{EF}$ & 04/10/91 & -5 & 5 & -4 & 0 & -5 & \\
\hline 205 & -10 & GW-197 & $\mathrm{EF}$ & 02/03/90 & -5 & 5 & -5 & 0 & -5 & \\
\hline 206 & -10 & GW-196 & $\mathrm{EF}$ & $02 / 21 / 90$ & -5 & 5 & -5 & 0 & -5 & \\
\hline 207 & -10 & GW-152 & $\mathrm{EF}$ & 02/28/90 & -5 & 5 & -5 & 0 & -5 & \\
\hline 208 & -10 & GW-268 & $\mathrm{EF}$ & 03/13/90 & -5 & 5 & -5 & 0 & -5 & \\
\hline 209 & -10 & GW-283 & $\mathrm{EF}$ & 10/14/93 & -5 & 5 & -5 & 0 & -5 & \\
\hline 210 & -10 & GW-239 & $\mathrm{EF}$ & 10/30/93 & -5 & 5 & -5 & 0 & -5 & \\
\hline 211 & -10 & GW-657 & $\mathrm{EF}$ & $11 / 15 / 93$ & -5 & 5 & -5 & 0 & -5 & \\
\hline 212 & -10 & GW-252 & $\mathrm{EF}$ & 05/17/95 & -5 & 5 & -5 & 0 & -5 & \\
\hline 213 & -10 & GW-255 & $\mathrm{EF}$ & 05/17/95 & -5 & 5 & -5 & 0 & -5 & \\
\hline 214 & -10 & GW-261 & $\mathrm{EF}$ & 05/16/95 & -5 & 5 & -5 & 0 & -5 & \\
\hline 215 & -10 & GW-263 & $\mathrm{EF}$ & 05/16/95 & -5 & 5 & -5 & 0 & -5 & \\
\hline 216 & -10 & GW-304 & $\mathrm{CR}$ & 07/12/95 & -5 & 5 & -5 & 0 & -5 & \\
\hline 217 & -10 & GW-303 & $\mathrm{CR}$ & 07/16/95 & -5 & 5 & -5 & 0 & -5 & \\
\hline 218 & -10 & GW-181 & $\mathrm{CR}$ & 11/06/95 & -5 & 5 & -5 & 0 & -5 & \\
\hline 219 & -10 & GW-511 & $\mathrm{CR}$ & $11 / 07 / 95$ & -5 & 5 & -5 & 0 & -5 & \\
\hline 220 & -10 & GW-759 & $\mathrm{EF}$ & $11 / 14 / 95$ & -5 & 5 & -5 & 0 & -5 & \\
\hline
\end{tabular}


Sampling Priority Scores for CY 2005

\begin{tabular}{|c|c|c|c|c|c|c|c|c|c|c|}
\hline \multirow[b]{2}{*}{ Rank } & \multirow{2}{*}{$\begin{array}{c}\text { Sampling } \\
\text { Priority } \\
\text { Score }^{1}\end{array}$} & \multirow[b]{2}{*}{ Well No. } & \multirow[b]{2}{*}{$\begin{array}{l}\text { Hydro. } \\
\text { Regime }^{2}\end{array}$} & \multirow{2}{*}{$\begin{array}{c}\text { Most } \\
\text { Recent } \\
\text { Sample }^{3}\end{array}$} & \multicolumn{5}{|c|}{ Points Scored ${ }^{4}$} & \multirow{2}{*}{$\begin{array}{l}\text { Selected for } \\
\text { CY } 2005^{5}\end{array}$} \\
\hline & & & & & Regime & $\begin{array}{c}\text { Time since } \\
\text { sampled last }\end{array}$ & $\begin{array}{c}\text { No. of } \\
\text { Samples }\end{array}$ & $\begin{array}{c}\text { Contaminant } \\
\text { Concentrations }\end{array}$ & $\begin{array}{l}\text { Trending } \\
\text { Potential }\end{array}$ & \\
\hline 221 & -10 & GW-199 & $\overline{E F}$ & $11 / 16 / 95$ & -5 & 5 & -5 & $\overline{0}$ & -5 & \\
\hline 222 & -10 & GW-773 & EF & $11 / 18 / 95$ & -5 & 5 & -5 & 0 & -5 & \\
\hline 223 & -10 & GW-774 & EF & $11 / 18 / 95$ & -5 & 5 & -5 & 0 & -5 & \\
\hline 224 & -10 & GW-546 & CR & 04/09/96 & -5 & 5 & -5 & 0 & -5 & \\
\hline 225 & -10 & GW-541 & $\mathrm{CR}$ & $04 / 15 / 96$ & -5 & 5 & -5 & 0 & -5 & \\
\hline 226 & -10 & GW-160 & CR & $04 / 29 / 96$ & -5 & 5 & -5 & 0 & -5 & \\
\hline 227 & -10 & GW-184 & $\mathrm{CR}$ & 04/30/96 & -5 & 5 & -5 & 0 & -5 & \\
\hline 228 & -10 & GW-186 & $\mathrm{CR}$ & 05/01/96 & -5 & 5 & -5 & 0 & -5 & \\
\hline 229 & -10 & GW-188 & CR & 04/30/96 & -5 & 5 & -5 & 0 & -5 & \\
\hline 230 & -10 & GW-298 & $\mathrm{CR}$ & 05/01/96 & -5 & 5 & -5 & 0 & -5 & \\
\hline 231 & -10 & GW-299 & $\mathrm{CR}$ & 04/30/96 & -5 & 5 & -5 & 0 & -5 & \\
\hline 232 & -10 & GW-512 & $\mathrm{CR}$ & $05 / 02 / 96$ & -5 & 5 & -5 & 0 & -5 & \\
\hline 233 & -10 & GW-292 & $\mathrm{CR}$ & 05/08/96 & -5 & 5 & -5 & 0 & -5 & \\
\hline 234 & -10 & GW-293 & CR & 05/08/96 & -5 & 5 & -5 & 0 & -5 & \\
\hline 235 & -10 & GW-766 & EF & $10 / 09 / 96$ & -5 & 5 & -5 & 0 & -5 & \\
\hline 236 & -10 & GW-767 & EF & $10 / 09 / 96$ & -5 & 5 & -5 & 0 & -5 & \\
\hline 237 & -10 & GW-191 & EF & $11 / 06 / 96$ & -5 & 5 & -5 & 0 & -5 & \\
\hline 238 & -10 & GW-194 & EF & $11 / 07 / 96$ & -5 & 5 & -5 & 0 & -5 & \\
\hline 239 & -10 & GW-195 & EF & $11 / 07 / 96$ & -5 & 5 & -5 & 0 & -5 & \\
\hline 240 & -10 & GW-779 & EF & $11 / 07 / 96$ & -5 & 5 & -5 & 0 & -5 & \\
\hline 241 & -10 & GW-780 & EF & $11 / 07 / 96$ & -5 & 5 & -5 & 0 & -5 & \\
\hline 242 & -10 & GW-149 & EF & $11 / 14 / 96$ & -5 & 5 & -5 & 0 & -5 & \\
\hline 243 & -10 & GW-751 & EF & $11 / 18 / 96$ & -5 & 5 & -5 & 0 & -5 & \\
\hline 244 & -10 & GW-752 & EF & $11 / 18 / 96$ & -5 & 5 & -5 & 0 & -5 & \\
\hline 245 & -10 & GW-384 & EF & $11 / 21 / 96$ & -5 & 5 & -5 & 0 & -5 & \\
\hline 246 & -10 & GW-385 & EF & $11 / 19 / 96$ & -5 & 5 & -5 & 0 & -5 & \\
\hline 247 & -10 & GW-603 & EF & $11 / 25 / 96$ & -5 & 5 & -5 & 0 & -5 & \\
\hline 248 & -10 & GW-817 & EF & $12 / 04 / 97$ & -5 & 5 & -5 & 0 & -5 & \\
\hline 249 & -10 & GW-745 & EF & $12 / 09 / 97$ & -5 & 5 & -5 & 0 & -5 & \\
\hline 250 & -10 & GW-746 & EF & $12 / 09 / 97$ & -5 & 5 & -5 & 0 & -5 & \\
\hline 251 & -10 & GW-748 & EF & $12 / 10 / 97$ & -5 & 5 & -5 & 0 & -5 & \\
\hline 252 & -10 & GW-749 & EF & $12 / 10 / 97$ & -5 & 5 & -5 & 0 & -5 & \\
\hline 253 & -10 & GW-754 & EF & 07/28/98 & -5 & 5 & -5 & 0 & -5 & \\
\hline 254 & -10 & GW-756 & EF & 07/27/98 & -5 & 5 & -5 & 0 & -5 & \\
\hline 255 & -10 & GW-753 & EF & 07/29/98 & -5 & 5 & -5 & 0 & -5 & \\
\hline 256 & -10 & GW-338 & EF & 08/20/98 & -5 & 5 & -5 & 0 & -5 & \\
\hline 257 & -10 & 1082 & $\mathrm{CR}$ & . & -5 & . & . & . & -5 & \\
\hline 258 & -10 & 1084 & CR & . & -5 & . & . & . & -5 & \\
\hline 259 & -10 & $55-2 A$ & EF & . & -5 & . & . & . & -5 & \\
\hline 260 & -10 & $55-3 A$ & EF & . & -5 & . & . & . & -5 & \\
\hline 261 & -10 & $55-3 B$ & EF & . & -5 & . & . & . & -5 & \\
\hline 262 & -10 & $56-1 A$ & EF & . & -5 & . & . & . & -5 & \\
\hline 263 & -10 & $56-3 A$ & EF & . & -5 & . & . & . & -5 & \\
\hline 264 & -10 & $56-3 B$ & EF & . & -5 & . & . & . & -5 & \\
\hline 265 & -10 & $56-4 A$ & EF & . & -5 & . & . & . & -5 & \\
\hline 266 & -10 & $56-6 A$ & EF & . & -5 & . & . & . & -5 & \\
\hline 267 & -10 & $56-8 A$ & EF & . & -5 & . & . & . & -5 & \\
\hline 268 & -10 & $58-2 A$ & EF & . & -5 & . & . & . & -5 & \\
\hline 269 & -10 & $60-1 A$ & EF & . & -5 & . & . & . & -5 & \\
\hline 270 & -10 & GW-558 & $\mathrm{CR}$ & . & -5 & . & . & . & -5 & \\
\hline 271 & -10 & GW-559 & $\mathrm{CR}$ & . & -5 & . & . & . & -5 & \\
\hline 272 & -10 & GW-674 & $\mathrm{CR}$ & . & -5 & . & . & . & -5 & \\
\hline 273 & -10 & GW-676 & $\mathrm{CR}$ & . & -5 & . & . & . & -5 & \\
\hline 274 & -10 & GW-677 & $\mathrm{CR}$ & . & -5 & . & . & . & -5 & \\
\hline 275 & -10 & GW-678 & $\mathrm{CR}$ & . & -5 & . & & . & -5 & \\
\hline
\end{tabular}




\section{Sampling Priority Scores for CY 2005}

\begin{tabular}{|c|c|c|c|c|c|c|c|c|c|c|}
\hline \multirow[b]{2}{*}{ Rank } & \multirow{2}{*}{$\begin{array}{c}\text { Sampling } \\
\text { Priority } \\
\text { Score }^{1}\end{array}$} & \multirow[b]{2}{*}{ Well No. } & \multirow{2}{*}{$\begin{array}{c}\text { Hydro. } \\
\text { Regime }^{2}\end{array}$} & \multirow{2}{*}{$\begin{array}{c}\text { Most } \\
\text { Recent } \\
\text { Sample }^{3}\end{array}$} & \multicolumn{5}{|c|}{ Points Scored ${ }^{4}$} & \multirow{2}{*}{$\begin{array}{l}\text { Selected for } \\
\text { CY } 2005^{5}\end{array}$} \\
\hline & & & & & Regime & $\begin{array}{c}\text { Time since } \\
\text { sampled last }\end{array}$ & $\begin{array}{c}\text { No. of } \\
\text { Samples }\end{array}$ & $\begin{array}{c}\text { Contaminant } \\
\text { Concentrations }\end{array}$ & $\begin{array}{l}\text { Trending } \\
\text { Potential }\end{array}$ & \\
\hline 276 & -10 & GW-734 & $\mathrm{EF}$ & & -5 & & & & -5 & \\
\hline 277 & -11 & GW-788 & $\mathrm{EF}$ & 10/20/99 & -5 & 4 & -5 & 0 & -5 & \\
\hline 278 & -11 & GW-781 & $\mathrm{EF}$ & $10 / 26 / 99$ & -5 & 4 & -5 & 0 & -5 & \\
\hline 279 & -11 & GW-792 & $\mathrm{EF}$ & $11 / 28 / 99$ & -5 & 4 & -5 & 0 & -5 & \\
\hline 280 & -13 & GW-609 & $\mathrm{CR}$ & 01/10/01 & -5 & 2 & -5 & 0 & -5 & \\
\hline 281 & -13 & GW-241 & $\mathrm{CR}$ & 08/16/01 & -5 & 2 & -5 & 0 & -5 & \\
\hline 282 & -13 & GW-514 & CR & 08/16/01 & -5 & 2 & -5 & 0 & -5 & \\
\hline 283 & -13 & GW-174 & $\mathrm{CR}$ & 08/21/01 & -5 & 2 & -5 & 0 & -5 & \\
\hline 284 & -13 & GW-608 & $\mathrm{CR}$ & 08/20/01 & -5 & 2 & -5 & 0 & -5 & \\
\hline 285 & -13 & GW-789 & $\mathrm{EF}$ & 10/15/01 & -5 & 2 & -5 & 0 & -5 & \\
\hline 286 & -13 & GW-349 & $\mathrm{EF}$ & $10 / 14 / 02$ & -5 & 1 & -4 & 0 & -5 & \\
\hline 287 & -13 & GW-350 & EF & $10 / 15 / 02$ & -5 & 1 & -4 & 0 & -5 & \\
\hline 288 & -14 & GW-539 & $\mathrm{CR}$ & $07 / 17 / 02$ & -5 & 1 & -5 & 0 & -5 & \\
\hline 289 & -14 & GW-776 & EF & $10 / 31 / 02$ & -5 & 1 & -5 & 0 & -5 & \\
\hline 290 & -15 & GW-302 & $\mathrm{CR}$ & 08/12/03 & -5 & 0 & -5 & 0 & -5 & \\
\hline 291 & -15 & GW-339 & $\mathrm{CR}$ & 08/11/03 & -5 & 0 & -5 & 0 & -5 & \\
\hline 292 & -15 & GW-142 & $\mathrm{CR}$ & 10/08/03 & -5 & 0 & -5 & 0 & -5 & \\
\hline 293 & -15 & GW-631 & EF & $10 / 07 / 03$ & -5 & 0 & -5 & 0 & -5 & \\
\hline 294 & -15 & GW-190 & EF & $10 / 21 / 03$ & -5 & 0 & -5 & 0 & -5 & \\
\hline 295 & -15 & GW-271 & $\mathrm{EF}$ & $10 / 20 / 03$ & -5 & 0 & -5 & 0 & -5 & \\
\hline 296 & -15 & GW-761 & EF & 10/29/03 & -5 & 0 & -5 & 0 & -5 & \\
\hline 297 & -15 & GW-300 & CR & $11 / 20 / 04$ & -5 & 0 & -5 & 0 & -5 & \\
\hline 298 & -15 & GW-513 & CR & $11 / 20 / 04$ & -5 & 0 & -5 & 0 & -5 & \\
\hline 299 & -15 & GW-610 & $\mathrm{CR}$ & $11 / 20 / 04$ & -5 & 0 & -5 & 0 & -5 & \\
\hline 300 & -15 & GW-611 & $\mathrm{CR}$ & $11 / 20 / 04$ & -5 & 0 & -5 & 0 & -5 & \\
\hline 301 & -15 & GW-742 & CR & $11 / 20 / 04$ & -5 & 0 & -5 & 0 & -5 & \\
\hline 302 & -15 & GW-743 & $\mathrm{CR}$ & $11 / 20 / 04$ & -5 & 0 & -5 & 0 & -5 & \\
\hline 303 & -15 & GW-760 & EF & $11 / 20 / 04$ & -5 & 0 & -5 & 0 & -5 & \\
\hline 304 & -15 & GW-765 & $\mathrm{EF}$ & $11 / 20 / 04$ & -5 & 0 & -5 & 0 & -5 & \\
\hline 305 & -15 & GW-786 & $\mathrm{EF}$ & $11 / 20 / 04$ & -5 & 0 & -5 & 0 & -5 & \\
\hline 306 & -15 & GW-787 & $\mathrm{EF}$ & $11 / 20 / 04$ & -5 & 0 & -5 & 0 & -5 & \\
\hline
\end{tabular}

Notes:

1 The Sampling Priority Score is the sum of the points scored.

$2 \mathrm{BC}=$ Bear Creek Hydrogeologic Regime

$\mathrm{CR}=$ Chestnut Ridge Hydrogeologic Regime

EF = Upper East Fork Poplar Creek Hydrogeologic Regime

3 Because sampling for CY 2004 is incomplete, the most recent sample dates for locations sampled during CY 2004 are default values based on the sampling schedule: first and third quarter locations use 08/15/03 as a default, and second and fourth quarter locations use 11/20/03. Some wells with relatively high ranking were not selected for CY 2004 because they were sampled within the previous year.

4 Point values are assigned in accordance with the monitoring optimization plan (see example on the following page).

$5 X=$ well selected for monitoring during CY 2005 (emphasized by shading the entire row). 


\section{Example of the CY 2005 sampling priority scoring: Well GW-097}

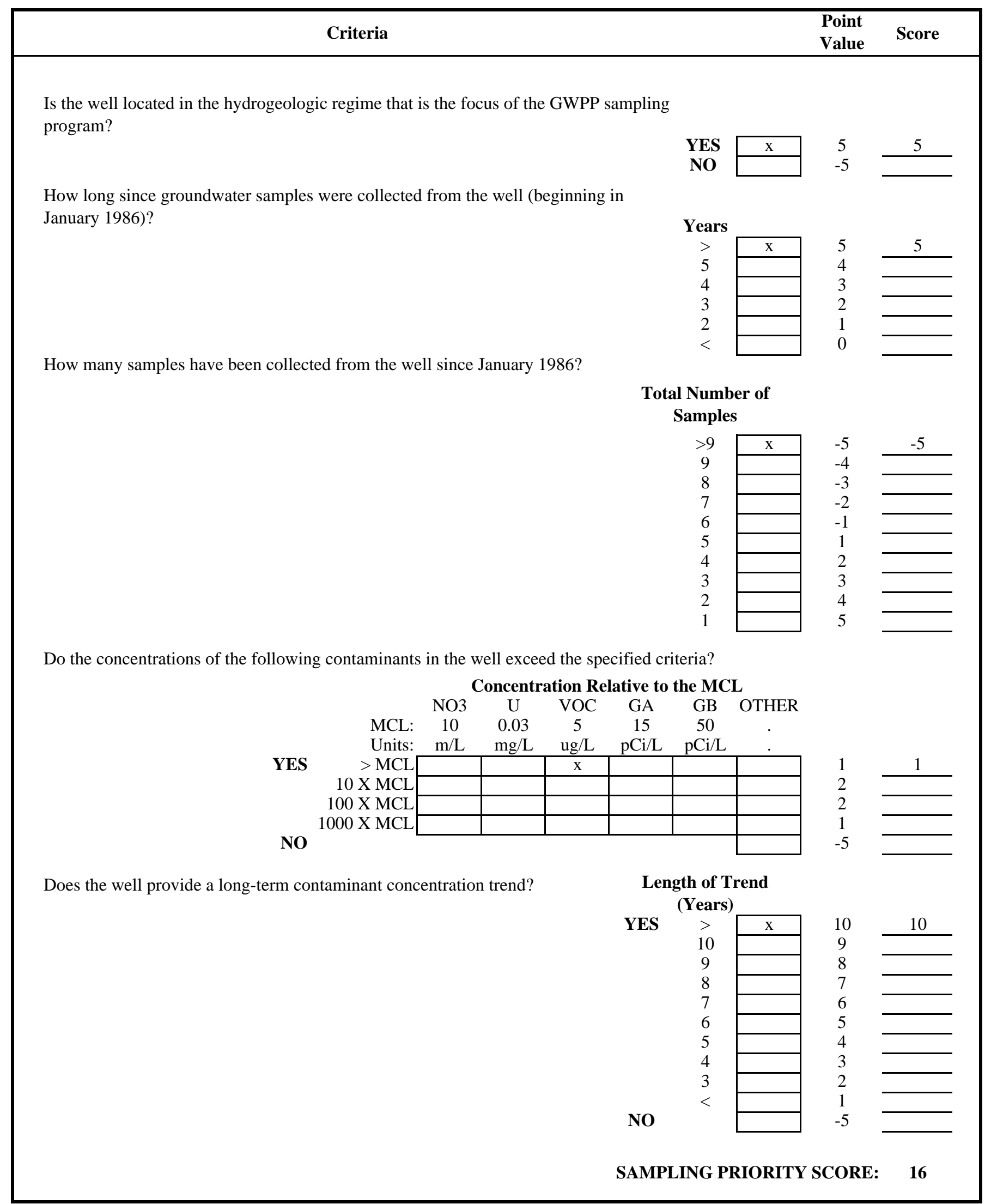


APPENDIX D

LABORATORY REQUIREMENTS

(Bottle Lists, Holding Times, Turnaround Time, Elevated Minimum Activity) 


\section{STD}

\begin{tabular}{|c|c|c|}
\hline Parameter & Chemical Preservative $^{1}$ & Bottle Types/Size \\
\hline Anions, Turbidity, and Fluoride & None & 1 - $250 \mathrm{~mL}$ polyethylene \\
\hline Total Suspended Solids & None & 1 - 250 mL polyethylene \\
\hline Total Dissolved Solids & None & 1 - $250 \mathrm{~mL}$ polyethylene \\
\hline Carbonate, Bicarbonate & None & 1 - 250 mL polyethylene \\
\hline $\begin{array}{l}\text { Total Metals (ICP,ICP-MS, and } \\
\mathrm{Hg})\end{array}$ & $\mathrm{HNO}_{3}$ & 1 - 500 mL polyethylene \\
\hline Radiochemistry (UV / Y-12) & $\mathrm{HNO}_{3}$ & 1 - $500 \mathrm{~mL}$ polyethylene \\
\hline VOA & None & $\begin{array}{l}2 \text { - } 40 \mathrm{~mL} \text { amber glass with } \\
\text { Teflon lined septum lids }\end{array}$ \\
\hline $\begin{array}{l}\text { Trip Blank (VOA) } \\
\text { (one per cooler) }\end{array}$ & None & $\begin{array}{l}1 \text { - } 40 \mathrm{~mL} \text { amber glass with } \\
\text { Teflon lined septum lid }\end{array}$ \\
\hline
\end{tabular}

STD:

FLD GWTRSAMP

ESLIMS LAB TEST ID

CHEM ALKALINITY-I, ANIONS, FLUORIDE, SOLIDS-TOT-S, SOLIDS-TOT-D, TURBIDITY

MET(1)ICP6010, ICPMSGW and HG7470

VOC(1) VOA8260GW

RAD(1)GROSSAB-ENV

${ }^{1}$ Samples chilled to $4+/-2 \mathrm{C}$ 


\section{STD, Rad (13)}

\begin{tabular}{||l|l|l||}
\hline \multicolumn{1}{|c|}{ Parameter } & \multicolumn{1}{c|}{ Chemical Preservative ${ }^{1}$} & \multicolumn{1}{c|}{ Bottle Types/Size } \\
\hline \hline Anions, Turbidity, and Fluoride & None & $1-250 \mathrm{~mL}$ polyethylene \\
\hline Total Suspended Solids & None & $1-250 \mathrm{~mL}$ polyethylene \\
\hline Total Dissolved Solids & $\mathrm{None}$ & $1-250 \mathrm{~mL}$ polyethylene \\
\hline Carbonate, Bicarbonate & $\mathrm{None}$ & $1-250 \mathrm{~mL}$ polyethylene \\
\hline $\begin{array}{l}\text { Total Metals (ICP,ICP-MS, and } \\
\text { Hg) }\end{array}$ & $\mathrm{HNO}_{3}$ & $1-500 \mathrm{~mL}$ polyethylene \\
\hline Radiochemistry (UV / Y-12) & $\mathrm{HNO}_{3}$ & $1-500 \mathrm{~mL}$ polyethylene \\
\hline TIMS & $\mathrm{HNO}_{3}$ & $1-250 \mathrm{~mL}$ polyethylene \\
\hline VOA & $\mathrm{None}^{2}$ & $\begin{array}{l}2-40 \mathrm{~mL} \text { amber glass with } \\
\text { Teflon lined septum lids }\end{array}$ \\
\hline $\begin{array}{l}\text { Trip Blank (VOA) } \\
\text { (one per cooler) }\end{array}$ & None & $\begin{array}{l}1-40 \mathrm{~mL} \text { amber glass with } \\
\text { Teflon lined septum lid }\end{array}$ \\
\hline
\end{tabular}

STD:

$\begin{array}{ll}\text { FLD } & \text { GWTRSAMP } \\ \text { CHEM } & \text { ALKALINITY-I, ANIONS, FLUORIDE, SOLIDS-TOT-S, } \\ & \text { SOLIDS-TOT-D, TURBIDITY }\end{array}$

MET(1)ICP6010, ICPMSGW and HG7470

VOC(1) VOA8260GW

RAD(1)GROSSAB-ENV

RAD(13) $\quad$ wt $\%{ }^{235} U$ and ug/g Total $U$ (TOTAL-U-ENV)

${ }^{1}$ Samples chilled to $4+/-2 \mathrm{C}$ 


\section{STD/F}

\begin{tabular}{|c|c|c|}
\hline Parameter & Chemical Preservative $^{1}$ & Bottle Types/Size \\
\hline Anions, Turbidity, and Fluoride & None & 1 - $250 \mathrm{~mL}$ polyethylene \\
\hline Total Suspended Solids & None & 1 - $250 \mathrm{~mL}$ polyethylene \\
\hline Total Dissolved Solids & None & 1 - $250 \mathrm{~mL}$ polyethylene \\
\hline Carbonate, Bicarbonate & None & 1 - $250 \mathrm{~mL}$ polyethylene \\
\hline $\begin{array}{l}\text { Total Metals (ICP,ICP-MS, and } \\
\mathrm{Hg} \text { ) }\end{array}$ & $\mathrm{HNO}_{3}$ & 1 - 500 mL polyethylene \\
\hline $\begin{array}{l}\text { Filtered Metals (ICP, ICP-MS, and } \\
\mathrm{Hg} \text { ) }\end{array}$ & $\mathrm{HNO}_{3}$ & 1 - $500 \mathrm{~mL}$ polyethylene \\
\hline Radiochemistry (UV / Y-12) & $\mathrm{HNO}_{3}$ & $1-500 \mathrm{~mL}$ polyethylene \\
\hline VOA & None & $\begin{array}{l}2 \text { - } 40 \mathrm{~mL} \text { amber glass with } \\
\text { Teflon lined septum lids }\end{array}$ \\
\hline $\begin{array}{l}\text { Trip Blank (VOA) } \\
\text { (one per cooler) }\end{array}$ & None & $\begin{array}{l}1 \text { - } 40 \mathrm{~mL} \text { amber glass with } \\
\text { Teflon lined septum lid }\end{array}$ \\
\hline
\end{tabular}

STD:

FLD

CHEM

$\operatorname{MET}(1) / F$

$\operatorname{VOC}(1)$

$\mathrm{RAD}(1)$
GWTRSAMP

ESLIMS LAB TEST ID

ALKALINITY-I, ANIONS, FLUORIDE, SOLIDS-TOT-S, TURBIDITY

SOLIDS-TOT-D

ICP6010, ICPMSGW and HG7470

VOA8260GW

GROSSAB-ENV

${ }^{1}$ Samples chilled to $4+/-2 \mathrm{C}$ 


\section{STD/F, Rad(13)}

\begin{tabular}{|c|c|c|}
\hline Parameter & Chemical Preservative $^{1}$ & Bottle Types/Size \\
\hline Anions, Turbidity, and Fluoride & None & 1 - $250 \mathrm{~mL}$ polyethylene \\
\hline Total Suspended Solids & None & 1 - $250 \mathrm{~mL}$ polyethylene \\
\hline Total Dissolved Solids & None & 1 - 250 mL polyethylene \\
\hline Carbonate, Bicarbonate & None & 1 - $250 \mathrm{~mL}$ polyethylene \\
\hline $\begin{array}{l}\text { Total Metals (ICP,ICP-MS, and } \\
\mathrm{Hg} \text { ) }\end{array}$ & $\mathrm{HNO}_{3}$ & 1 - 500 mL polyethylene \\
\hline $\begin{array}{l}\text { Filtered Metals (ICP, ICP-MS, and } \\
\mathrm{Hg})\end{array}$ & $\mathrm{HNO}_{3}$ & 1 - 500 mL polyethylene \\
\hline Radiochemistry (UV / Y-12) & $\mathrm{HNO}_{3}$ & 1 - $500 \mathrm{~mL}$ polyethylene \\
\hline TIMS & $\mathrm{HNO}_{3}$ & $1-250 \mathrm{~mL}$ polyethylene \\
\hline VOA & None & $\begin{array}{l}2 \text { - } 40 \mathrm{~mL} \text { amber glass with } \\
\text { Teflon lined septum lids }\end{array}$ \\
\hline $\begin{array}{l}\text { Trip Blank (VOA) } \\
\text { (one per cooler) }\end{array}$ & None & $\begin{array}{l}1 \text { - } 40 \mathrm{~mL} \text { amber glass with } \\
\text { Teflon lined septum lid }\end{array}$ \\
\hline
\end{tabular}

STD:

FLD

GWTRSAMP

CHEM ALKALINITY-I, ANIONS, FLUORIDE, SOLIDS-TOT-S, TURBIDITY SOLIDS-TOT-D

MET(1)/F ICP6010, ICPMSGW and HG7470

VOC(1) VOA8260GW

RAD(1)GROSSAB-ENV

$\mathrm{RAD}(13) \quad \mathrm{wt} \%{ }^{235} \mathrm{U}$ and $\mathrm{ug} / \mathrm{g}$ Total $\mathrm{U}$ (TOTAL-U-ENV)

${ }^{1}$ Samples chilled to $4+/-2 \mathrm{C}$ 


\section{$\operatorname{VOC}(1)$}

\begin{tabular}{||c|c|c||}
\hline \multicolumn{1}{|c|}{ Parameter } & Chemical Preservative $^{1}$ & Bottle Types/Size $^{-1}$ \\
\hline \hline VOA & None & $\begin{array}{l}2-40 \mathrm{~mL} \text { amber glass with } \\
\text { Teflon lined septum lid }\end{array}$ \\
\hline
\end{tabular}

$\operatorname{VOC}(1)$

VOA8260GW

${ }^{1}$ Samples chilled to $4+/-2 \mathrm{C}$ 


\section{STD, RAD (3)}

\begin{tabular}{||l|l|l||}
\hline \multicolumn{1}{|c|}{ Parameter } & \multicolumn{1}{c||}{ Chemical Preservative $^{1}$} & \multicolumn{1}{c|}{ Bottle Types/Size $^{-1}$} \\
\hline \hline Anions, Turbidity, and Fluoride & None & $1-250 \mathrm{~mL}$ polyethylene \\
\hline Total Suspended Solids & None & $1-250 \mathrm{~mL}$ polyethylene \\
\hline Total Dissolved Solids & None & $1-250 \mathrm{~mL}$ polyethylene \\
\hline Carbonate, Bicarbonate & None & $1-250 \mathrm{~mL}$ polyethylene \\
\hline $\begin{array}{l}\text { Total Metals (ICP,ICP-MS, and } \\
\text { Hg) }\end{array}$ & $\mathrm{HNO}_{3}$ & $1-500 \mathrm{~mL}$ polyethylene \\
\hline Radiochemistry (UV / Y12) & $\mathrm{HNO}_{3}$ & $1-1 \mathrm{~L}$ polyethylene \\
\hline $\begin{array}{l}\text { VOA } \\
\text { None }\end{array}$ & $\begin{array}{l}2-40 \mathrm{~mL} \text { amber glass with } \\
\text { Teflon lined septum lids }\end{array}$ \\
\hline $\begin{array}{l}\text { Trip Blank (VOA) } \\
\text { (one per cooler) }\end{array}$ & None & $\begin{array}{l}1-40 \mathrm{~mL} \text { amber glass with } \\
\text { Teflon lined septum lid }\end{array}$ \\
\hline
\end{tabular}

$\begin{array}{llll}\text { STD: } & \text { ESLIMS LAB TEST ID } & \\ \text { FLD } & \text { GWSPSAMP } \\ \text { CHEM } & \text { ALKALINITY-I, ANIONS, FLUORIDE, SOLIDS-TOT-S, } & \\ & \text { SOLIDS-TOT-D, TURBIDITY } & \\ \text { MET(1)ICP6010, ICPMSGW and HG7470 } \\ \text { VOC(1) } & \text { VOA8260GW } \\ & & \\ \text { RAD (1) } & \text { GrosS Alpha Beta (GROSSAB-ENV) } & 500 \mathrm{~mL} & \text { preserved } \mathrm{w} \mathrm{HNO}_{3} \\ \text { RAD (3) } & { }^{234} \mathrm{U},{ }^{235} \mathrm{U},{ }^{238} \mathrm{U} \text { (ASPECU-ENV) } & 500 \mathrm{~mL} & \text { preserved } \mathrm{wNO}_{3}\end{array}$

${ }^{1}$ Samples chilled to $4+/-2 C$ 


\section{STD, RAD $(3,13)$}

\begin{tabular}{||l|l|l||}
\hline \multicolumn{1}{|c|}{ Parameter } & Chemical Preservative $^{1}$ & \multicolumn{1}{c||}{ Bottle Types/Size $^{-1}$} \\
\hline \hline Anions, Turbidity, and Fluoride & None & $1-250 \mathrm{~mL}$ polyethylene \\
\hline Total Suspended Solids & None & $1-250 \mathrm{~mL}$ polyethylene \\
\hline Total Dissolved Solids & None & $1-250 \mathrm{~mL}$ polyethylene \\
\hline Carbonate, Bicarbonate & None & $1-250 \mathrm{~mL}$ polyethylene \\
\hline $\begin{array}{l}\text { Total Metals (ICP,ICP-MS, and } \\
\text { Hg) }\end{array}$ & $\mathrm{HNO}_{3}$ & $1-500 \mathrm{~mL}$ polyethylene \\
\hline Radiochemistry (UV/Y12) & $\mathrm{HNO}_{3}$ & $1-1 \mathrm{~L}$ polyethylene \\
\hline TIMS & $\mathrm{HNO}_{3}$ & $1-250 \mathrm{~mL}$ polyethylene \\
\hline VOA & $\mathrm{None}_{2}$ & $\begin{array}{l}2-40 \mathrm{~mL} \text { amber glass with } \\
\text { Teflon lined septum lids }\end{array}$ \\
\hline $\begin{array}{l}\text { Trip Blank (VOA) } \\
\text { (one per cooler) }\end{array}$ & None & $\begin{array}{l}1-40 \mathrm{~mL} \text { amber glass with } \\
\text { Teflon lined septum lid }\end{array}$ \\
\hline
\end{tabular}

STD: $\quad$ ESLIMS LAB TEST ID

FLD GWSPSAMP

CHEM ALKALINITY-I, ANIONS, FLUORIDE, SOLIDS-TOT-S, SOLIDS-TOT-D, TURBIDITY

MET(1)ICP6010, ICPMSGW and HG7470

$\operatorname{VOC}(1) \quad$ VOA8260GW

RAD (1) Gross Alpha Beta (GROSSAB-ENV) $500 \mathrm{~mL}$ preserved w $\mathrm{HNO}_{3}$

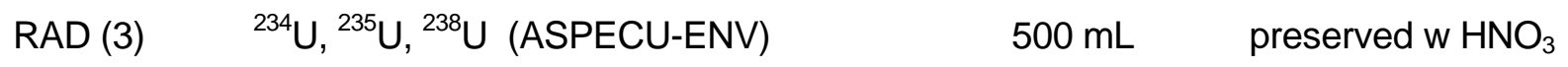

RAD(13) $\quad w t \%{ }^{235} \mathrm{U}$ and ug/g Total $U$ (TOTAL-U-ENV) $250 \mathrm{~mL} \quad$ preserved $w \mathrm{HNO}_{3}$

\footnotetext{
${ }^{1}$ Samples chilled to $4+/-2 \mathrm{C}$
} 


\section{STD, RAD (12)}

\begin{tabular}{||l|l|l|}
\hline \multicolumn{1}{|c|}{ Parameter } & \multicolumn{1}{c|}{ Chemical Preservative $^{1}$} & \multicolumn{1}{c|}{ Bottle Types/Size $^{-1}$} \\
\hline \hline Anions, turbidity, and Fluoride & None & $1-250 \mathrm{~mL}$ polyethylene \\
\hline Total Suspended Solids & None & $1-250 \mathrm{~mL}$ polyethylene \\
\hline Total Dissolved Solids & None & $1-250 \mathrm{~mL}$ polyethylene \\
\hline Carbonate, Bicarbonate & None & $1-250 \mathrm{~mL}$ polyethylene \\
\hline $\begin{array}{l}\text { Total Metals (ICP,ICP-MS, and } \\
\text { Hg) }\end{array}$ & $\mathrm{HNO}_{3}$ & $1-500 \mathrm{~mL}$ polyethylene \\
\hline Radiochemistry (UV / Y12) & $\mathrm{HNO}_{3}$ & $1-$ liter polyethylene \& \\
\hline $\begin{array}{l}\text { VOA } \\
\text { None }\end{array}$ & $\begin{array}{l}2-40 \mathrm{~mL} \text { amber glass with } \\
\text { Teflon lined septum lids }\end{array}$ \\
\hline $\begin{array}{l}\text { Trip Blank (VOA) } \\
\text { (one per cooler) }\end{array}$ & $\begin{array}{l}1-40 \mathrm{~mL} \text { amber glass with } \\
\text { Teflon lined septum lid }\end{array}$ \\
\hline
\end{tabular}

\begin{tabular}{ll}
\hline STD: & ESLIMS LAB TEST ID \\
FLD & GWTRSAMP \\
CHEM & ALKALINITY-I, ANIONS, FLUORIDE, SOLIDS-TOT-S, \\
& SOLIDS-TOT-D, TURBIDITY \\
MET(1)ICP6010, ICPMSGW and HG7470 \\
VOC(1) & VOA8260GW
\end{tabular}

RAD (1) Gross Alpha Beta (GROSSAB-ENV) $500 \mathrm{~mL} \quad$ preserved w $\mathrm{HNO}_{3}$

RAD (12) Tc-99 (TC99LS-ENV) $500 \mathrm{~mL} \quad$ preserved $\mathrm{w} \mathrm{HNO}_{3}$

${ }^{1}$ Samples chilled to $4+/-2 \mathrm{C}$ 


\section{STD, RAD $(12,13)$}

\begin{tabular}{|c|c|c|}
\hline Parameter & Chemical Preservative $^{1}$ & Bottle Types/Size \\
\hline Anions, turbidity, and Fluoride & None & 1 - $250 \mathrm{~mL}$ polyethylene \\
\hline Total Suspended Solids & None & $1-250 \mathrm{~mL}$ polyethylene \\
\hline Total Dissolved Solids & None & $1-250 \mathrm{~mL}$ polyethylene \\
\hline Carbonate, Bicarbonate & None & $1-250 \mathrm{~mL}$ polyethylene \\
\hline $\begin{array}{l}\text { Total Metals (ICP,ICP-MS, and } \\
\mathrm{Hg} \text { ) }\end{array}$ & $\mathrm{HNO}_{3}$ & $1-500 \mathrm{~mL}$ polyethylene \\
\hline Radiochemistry (UV / Y12) & $\mathrm{HNO}_{3}$ & 1 - liter polyethylene \& \\
\hline TIMS & $\mathrm{HNO}_{3}$ & $1-250 \mathrm{~mL}$ polyethylene \\
\hline VOA & None & $\begin{array}{l}2-40 \mathrm{~mL} \text { amber glass with } \\
\text { Teflon lined septum lids }\end{array}$ \\
\hline $\begin{array}{l}\text { Trip Blank (VOA) } \\
\text { (one per cooler) }\end{array}$ & None & $\begin{array}{l}1-40 \mathrm{~mL} \text { amber glass with } \\
\text { Teflon lined septum lid }\end{array}$ \\
\hline
\end{tabular}

\begin{tabular}{ll}
\hline STD: & ESLIMS LAB TEST ID \\
FLD & GWTRSAMP \\
CHEM & ALKALINITY-I, ANIONS, FLUORIDE, SOLIDS-TOT-S, \\
& SOLIDS-TOT-D, TURBIDITY \\
MET(1)ICP6010, ICPMSGW and HG7470 \\
VOC(1) & VOA8260GW
\end{tabular}

RAD (1) Gross Alpha Beta (GROSSAB-ENV) $500 \mathrm{~mL} \quad$ preserved w $\mathrm{HNO}_{3}$

RAD (12) TC-99 (TC99LS-ENV) $500 \mathrm{~mL} \quad$ preserved $\mathrm{w} \mathrm{HNO}_{3}$

$\mathrm{RAD}(13) \quad \mathrm{wt} \%{ }^{235} \mathrm{U}$ and $\mathrm{ug} / \mathrm{g}$ Total $\mathrm{U}$ (TOTAL-U-ENV) $250 \mathrm{~mL} \quad$ preserved $\mathrm{w} \mathrm{HNO}_{3}$

${ }^{1}$ Samples chilled to $4+/-2 \mathrm{C}$ 


\section{STD, RAD $(3,12,13)$}

\begin{tabular}{||l|l|l||}
\hline \multicolumn{1}{|c|}{ Parameter } & Chemical Preservative $^{1}$ & \multicolumn{1}{c|}{ Bottle Types/Size $^{-1}$} \\
\hline \hline Anions, Turbidity, and Fluoride & None & $1-250 \mathrm{~mL}$ polyethylene \\
\hline Total Suspended Solids & None & $1-250 \mathrm{~mL}$ polyethylene \\
\hline Total Dissolved Solids & None & $1-250 \mathrm{~mL}$ polyethylene \\
\hline Carbonate, Bicarbonate & None & $1-250 \mathrm{~mL}$ polyethylene \\
\hline $\begin{array}{l}\text { Total Metals (ICP,ICP-MS, and } \\
\text { Hg) }\end{array}$ & $\mathrm{HNO}_{3}$ & $1-500 \mathrm{~mL}$ polyethylene \\
\hline Radiochemistry (UV/Y12) & $\mathrm{HNO}_{3}$ & $\begin{array}{l}1-\text { liter polyethylene \& } \\
1-500 \mathrm{~mL} \text { polyethylene }\end{array}$ \\
\hline TIMS & $\mathrm{HNO}_{3}$ & $1-250-\mathrm{mL}$ polyethylene \\
\hline VOA & None & $\begin{array}{l}2-40 \mathrm{~mL} \text { amber glass with } \\
\text { Teflon lined septum lids }\end{array}$ \\
\hline $\begin{array}{l}\text { Trip Blank (VOA) } \\
\text { (one per cooler) }\end{array}$ & None & $\begin{array}{l}1-40 \mathrm{~mL} \text { amber glass with } \\
\text { Teflon lined septum lid }\end{array}$ \\
\hline
\end{tabular}

\begin{tabular}{|c|c|c|c|}
\hline $\begin{array}{l}\text { STD: } \\
\text { FLD } \\
\text { CHEM }\end{array}$ & $\begin{array}{l}\text { ESLIMS LAB TEST ID } \\
\text { GWTRSAMP } \\
\text { ALKALINITY-I, ANIONS, FLUORIDE } \\
\text { SOLIDS-TOT-D, TURBIDITY }\end{array}$ & S-TOT-S, & \\
\hline $\begin{array}{l}\operatorname{MET}(1)|C| \\
\operatorname{VOC}(1)\end{array}$ & \multicolumn{3}{|l|}{ VOA8260GW } \\
\hline RAD (1) & Gross Alpha Beta (GROSSAB-ENV) & $500 \mathrm{~mL}$ & preserved $\mathrm{W} \mathrm{HNO}_{3}$ \\
\hline RAD (3) & ${ }^{234} U,{ }^{235} U,{ }^{238} U$ (ASPECU-ENV) & $500 \mathrm{~mL}$ & preserved $\mathrm{w} \mathrm{HNO}_{3}$ \\
\hline RAD (12) & Tc-99 (TC99LS-ENV) & $500 \mathrm{~mL}$ & preserved $\mathrm{w} \mathrm{HNO}_{3}$ \\
\hline $\operatorname{RAD}(13)$ & $\mathrm{wt} \%{ }^{235} \mathrm{U}$ and $\mathrm{ug} / \mathrm{g}$ Total $\mathrm{U}$ (TOTAL- & $250 \mathrm{~mL}$ & preserved $\mathrm{w} \mathrm{HNO}_{3}$ \\
\hline
\end{tabular}

${ }^{1}$ Samples chilled to $4+/-2 \mathrm{C}$ 


\section{WESTBAY}

\begin{tabular}{||l|l|l||}
\hline \multicolumn{1}{|c|}{ Parameter } & \multicolumn{1}{c||}{ Chemical Preservative ${ }^{1}$} & \multicolumn{1}{c|}{ Bottle Types/Size $^{-1}$} \\
\hline \hline Anions, Turbidity, and Fluoride & None & $1-250 \mathrm{~mL}$ polyethylene \\
\hline Total Suspended Solids & None & $1-250 \mathrm{~mL}$ polyethylene \\
\hline Total Dissolved Solids & None & $1-250 \mathrm{~mL}$ polyethylene \\
\hline Carbonate, Bicarbonate & $\mathrm{None}$ & $1-250 \mathrm{~mL}$ polyethylene \\
\hline $\begin{array}{l}\text { Total Metals (ICP,ICP-MS, and } \\
\text { Hg) }\end{array}$ & $\mathrm{HNO}_{3}$ & $1-250 \mathrm{~mL}$ polyethylene \\
\hline Radiochemistry (UV / Y-12) & $\mathrm{HNO}_{3}$ & $1-250 \mathrm{~mL}$ polyethylene \\
\hline $\begin{array}{l}\text { VOA } \\
\mathrm{None}^{\prime}\end{array}$ & $\begin{array}{l}2-40 \mathrm{~mL} \text { amber glass with } \\
\text { Teflon lined septum lids }\end{array}$ \\
\hline $\begin{array}{l}\text { Trip Blank (VOA) } \\
\text { (one per cooler) }\end{array}$ & None & $\begin{array}{l}1-40 \mathrm{~mL} \text { amber glass with } \\
\text { Teflon lined septum lid }\end{array}$ \\
\hline
\end{tabular}

STD:

FLD

GWTRSAMP

ESLIMS LAB TEST ID

CHEM ALKALINITY-I, ANIONS, FLUORIDE, SOLIDS-TOT-S, SOLIDS-TOT-D, TURBIDITY

MET(1)ICP6010, ICPMSGW and HG7470

VOC(1) VOA8260GW

RAD(1)GROSSAB-ENV

${ }^{1}$ Samples chilled to $4+/-2 \mathrm{C}$ 


\section{ESTABLISHED HOLDING TIMES}

\begin{tabular}{|l|l|}
\hline Parameter & Holding Times \\
\hline Alkalinity (Carbonate, Bicarbonate) & 14 days \\
\hline Anions (Chloride, Nitrate, Sulfate) & 48 hr \\
\hline Fluoride & 28 days \\
\hline Mercury & 28 days \\
\hline Metals (ICP, ICPMS) & 6 months \\
\hline Radiochemistry (except tritium) & 6 months \\
\hline Solids, Total Dissolved & 7 days \\
\hline Solids, Total Suspended & 7 days \\
\hline Tritium & No EPA guidance \\
\hline Uranium by Thermal Ionization Mass Spec & 6 months \\
\hline VOA & 7 days \\
\hline
\end{tabular}

\section{ESTABLISHED TURNAROUND TIMES}

The Groundwater Protection Program and the Analytical Chemistry Organization (ACO) laboratory have agreed upon a turnaround time, such that the analytical data generated from all sample locations within a sample group will be transmitted to the Data Manager as a data deliverable. Currently, the turnaround time for all sample groups is 35 days from the receipt of the last sample within a group. Data is transmitted in the form of hard copy of the completed and approved lab reports for each locations, along with an electronic copy in a standardized and compatible format (please see Y-12 Plant Groundwater Protection Program Data Management Plan, Revision 2, November 2003, Y/SUB/03-013288/1).

\section{ELEVATED MINIMUM DETECTABLE ACTIVITY}

Groundwater samples with high TDS (>1,000 mg/L) typically have elevated minimum detectable activities (MDAs) for gross alpha (>15 pCi/L) and gross beta (> $50 \mathrm{pCi} / \mathrm{L})$. However, the MDAs for specific isotopic analyses are unaffected by the sample solid content. For samples with gross activity results that are less than an elevated MDA, and specific isotopic analyses have not been requested, the laboratory will issue a request to analyze for the principal alpha- or beta-emitting isotopes. That is, if the gross alpha MDA exceeds $15 \mathrm{pCi} / \mathrm{L}$ and the result is less than $15 \mathrm{pCi} / \mathrm{L}$, then the laboratory will request analyses of isotopic uranium (by method Y/P65-7061). Similarly, if a sample has an elevated gross beta MDA ( $>50 \mathrm{pCi} / \mathrm{L})$ and the result is less than the MDA, then the laboratory would request analysis of technetium-99 activity. These requests will be approved by the Y-12 Groundwater Protection Program manager, or designee, before analyses are performed. 
APPENDIX E

ADDENDA TO THE CY 2005 SAMPLING AND ANALYSIS PLAN (if issued) 
APPENDIX F

CY 2004 GROUNDWATER MONITORING SCHEDULES 


\section{DISTRIBUTION}

\section{U.S. DEPARTMENT OF ENERGY}

J.P. Donnelly

ENVIRONMENTAL COMPLIANCE

DEPARTMENT

J.M. Eaton

S.M. Field

S.B. Jones

K.G. Hanzelka

C.C. Hill

E.R. Schultz

L.O. Vaughan

GWPP-File (2)

EC Document Center

\section{ANALYTICAL CHEMISTRY}

ORGANIZATION

D.D. Altom

L.E. White (2)

TECHNICAL COMPUTING

S.W. King

YDCC - RC

Y-12 Central Files

Y-12 Records Services (Electronic copy-OSTI)

\section{ELVADO ENVIRONMENTAL LLC}

T.R. Harrison

J.R. Walker

SCIENCE APPLICATIONS INTERNATIONAL CORPORATION

J.L. Vance

W.K. Jago

D.L. Smith

BECHTEL JACOBS COMPANY LLC

E.L. Berglund

J.I. Cox

H.L. King

L.M. Sims (2)

File-EMEF-DMC

COMMODORE ADVANCED SCIENCES, INC.

W. Foutz

UT-BATTELLE, LLC

D.B. Watson

TENNESSEE DEPARTMENT OF ENVIRONMENT AND CONSERVATION DOE OVERSIGHT

D. Gilmore (3) 